\title{
On exact overlaps in integrable spin chains
}

\section{Yunfeng Jiang ${ }^{a}$ and Balázs Pozsgay ${ }^{b}$}

${ }^{a}$ Theoretical Physics Department, CERN, Geneva, Switzerland

${ }^{b}$ MTA-BME Quantum Dynamics and Correlations Research Group, Department of Theoretical Physics, Budapest University of Technology and Economics, 1521 Budapest, Hungary

E-mail: jinagyf2008@gmail.com, pozsgay.balazs@gmail.com

ABSTRACT: We compute the exact overlaps between integrable boundary states and onshell Bethe states for integrable spin chains. Our method is based on the coordinate Bethe Ansatz and does not rely on the "rotation trick" of the corresponding lattice model. It leads to a rigorous proof of the factorized overlap formulae in a number of cases, some of which were hitherto inaccessible to earlier methods. As concrete examples, we consider the compact XXX and XXZ Heisenberg spin chains, and the non-compact $\mathrm{SL}(2, \mathbb{R})$ spin chain.

Keywords: Bethe Ansatz, Lattice Integrable Models, Boundary Quantum Field Theory ARXIV EPRINT: 2002.12065 


\section{Contents}

1 Introduction 1

2 Integrable local spin chains and Bethe Ansatz 3

2.1 Local integrable spin chains 3

2.2 Coordinate Bethe Ansatz 5

3 Integrable boundary states $\quad 7$

3.1 General discussion 8

$\begin{array}{ll}3.2 \text { The compact chains } & 10\end{array}$

$\begin{array}{ll}3.3 \text { The non-compact chain } & 11\end{array}$

$\begin{array}{ll}3.4 \text { Exact overlap formulae } & 15\end{array}$

4 Exact overlap formulae - General strategy $\quad 17$

$\begin{array}{ll}4.1 \text { One-particle states } & 18\end{array}$

$\begin{array}{lll}4.2 & \text { Two-particle states } & 18\end{array}$

4.3 Multi-particle states 20

5 Exact overlap formulae - Concrete cases $\quad 24$

5.1 The state $\left|X_{F}\right\rangle$ in the Heisenberg chains 24

$\begin{array}{ll}\text { 5.1.1 Determining the singular piece } & 26\end{array}$

5.2 Néel and generalized Néel states in the Heisenberg chains 28

5.3 Generalized Néel state in the $\mathrm{SL}(2, \mathbb{R})$ chain $\quad 29$

6 Conclusions and discussions $\quad 30$

$\begin{array}{ll}\text { A Formula for coherent states } & 31\end{array}$

\section{Introduction}

The overlap between an integrable boundary state and an on-shell energy eigenstate is an important quantity in integrable models. In integrable quantum field theories, when the energy eigenstate is the ground state, the overlap is known as the exact $g$-function. The $g$ function is a measure of boundary degrees of freedom and is thus also called the boundary entropy. Very recently, this quantity made its appearance in the context of AdS/CFT where it is shown $[1,2]$ that the structure constant of two determinant operators and one non-BPS single trace operator at finite coupling is given by an exact $g$-function on the string world sheet.

Turning to integrable lattice models such as integrable quantum spin chains and classical statistical lattice models, these overlaps also play an important role. They are crucial 
ingredients in the context of quantum quenches, partition functions of integrable lattice models [3], as well as the weak coupling limit of integrability in AdS/CFT [4-6].

The first exact result for on-shell overlaps appeared in [7] based on the earlier works [8, 9]. It was found that only the Bethe states whose rapidities are parity symmetric lead to non-vanishing overlaps. This finding was explained in [10], where an integrability condition was formulated for the boundary states. It was further argued in [11] that it is only these integrable states where simple factorized results can be expected. This expectation was confirmed in all known cases (see [12] and references therein). It is now understood that the integrable boundary states are closely connected to integrable boundary conditions $[10,11$, 13], generalizing the seminal results of Ghoshal and Zamolodchikov on integrable boundary QFT [14].

The exact finite volume overlap formulae have the same structure in all known cases: they are given by a product of two parts. One part is universal and is given by the ratio of two so-called Gaudin like determinants (which are replaced by Fredholm determinants in the continuum limit or in the AdS/CFT situation). The other part depends on the details of the boundary state and is a product of simple scalar factors, or a sum of such products. We note that the first work which derived this structure was [15], although the early results of [15] only pertained to integrable QFT and they were not used in the later studies of the spin chain overlaps.

The works mentioned above concern compact spin chains, where the quantum space at each site is finite dimensional. On the other hand, non-compact chains with infinite dimensional local Hilbert spaces are highly relevant in QCD and AdS/CFT. To the best of our knowledge, integrable boundary states of non-compact spin chains have never been studied before. Recently, an exact overlap formula with a specific boundary state in a noncompact chain was conjectured [1] in the context of AdS/CFT. The factorized overlap takes the same form as in the compact case. In the present paper we show that this boundary state is indeed integrable, and provide an actual proof for the conjectured overlap formula.

We stress that up to now there have been no methods to actually prove the exact overlap formulae, except for the simplest cases in the Heisenberg spin chains which are related to the so-called diagonal $K$-matrices [7]. The proof of [7] uses an off-shell overlap formula, which goes back to the work of Tsushiya [16] (see also [8, 9]). It is most likely that such an off-shell formula does not exist in other cases, which are related to off-diagonal $K$-matrices in the XXZ chain, or any $K$-matrix in higher rank cases. The follow-up works assumed that the structure of the factorized overlap is the same in all cases, and determined the one-particle overlap functions using a generalization of the Quantum Transfer Matrix (QTM) method [11,12]. Alternatively, the one-particle overlap functions could be extracted from coordinate Bethe Ansatz computations [4-6]. And while QTM approach was rather successful in the compact spin chain, it is not evident whether it can be generalized to the non-compact cases.

In this work we go back to the coordinate Bethe Ansatz solution of the models, and rigorously prove the overlap formulae starting from the explicit finite volume wave functions. Our approach is a combination of the real space methods of $[4,5]$ and some ideas present in Korepin's work [17], where it was rigorously proven that the norm of the on-shell 
Bethe states is given by the Gaudin determinant. Our main idea is to focus on the apparent singularities of the overlaps, but instead of developing a completely algebraic approach we compute the singularities from coordinate Bethe Ansatz.

The rest of the paper is structured as follows. In section 2 we introduce the local spin chains that we study in this paper and review their solution by coordinate Bethe Ansatz. In section 3 we discuss integrable boundary states for these spin chains. We also prove the boundary state proposed in [1] is indeed integrable. We give the general strategy for the proof of exact overlap formulae using coordinate Bethe Ansatz in section 4. Concrete examples for both compact and non-compact spin chains are presented in section 5 . We conclude and discuss some future directions in section 6 .

\section{$2 \quad$ Integrable local spin chains and Bethe Ansatz}

We review the definitions of various local integrable quantum spin chains and their solutions by Bethe Ansatz. More specifically, we will consider the compact XXX and XXZ spin chains and the non-compact $\mathrm{SL}(2, \mathbb{R})$ spin chain.

\subsection{Local integrable spin chains}

We consider integrable spin chains given by local Hamiltonians

$$
H=\sum_{j=1}^{L} h_{j, j+1}
$$

with periodic boundary condition. We denote the Hilbert space of each local site $j$ by $\mathcal{H}_{j}$. The dimension of $\mathcal{H}_{j}$ can be finite or infinite. Each term $h_{j, j+1}$ act on the space $\mathcal{H}_{j} \otimes \mathcal{H}_{j+1}$.

Compact spin chain. The Hamiltonian for the compact XXZ spin chain is given by

$$
H=\sum_{j=1}^{L}\left(\sigma_{j}^{x} \sigma_{j+1}^{x}+\sigma_{j}^{y} \sigma_{j+1}^{y}+\Delta\left(\sigma_{j}^{z} \sigma_{j+1}^{z}-1\right)\right) .
$$

where $\sigma_{j}^{\alpha}(\alpha=x, y, z)$ are the Pauli matrices. Here $\Delta$ is the anisotropy parameter. The isotropic XXX spin chain corresponds to taking $\Delta=1$. For simplicity we focus on the so-called massive regime $\Delta \geq 1$ for XXZ spin chain in this paper.

The local Hilbert space at each site is $\mathbb{C}^{2}$. The two basis vectors are

$$
|\uparrow\rangle=\left(\begin{array}{l}
1 \\
0
\end{array}\right), \quad|\downarrow\rangle=\left(\begin{array}{l}
0 \\
1
\end{array}\right) .
$$

The isotropic XXX spin chain has SU(2) symmetry. The local Hilbert spaces form the the spin- $\frac{1}{2}$ representation of the $\mathfrak{s u}(2)$ algebra.

Non-compact spin chain. Now we consider the non-compact $\operatorname{SL}(2, \mathbb{R})$ spin chain. ${ }^{1}[18$, 19]. We first introduce the $\operatorname{SL}(2, \mathbb{R})$ algebra. The generators in the spin- $s$ representation

\footnotetext{
${ }^{1}$ This spin chain is nothing but the Heisenberg $\mathrm{XXX}_{s}$ spin chain with local quantum space in the noncompact $s=-1 / 2$ representation. We choose to call it the $\operatorname{SL}(2, \mathbb{R})$ spin chain in accordance with the QCD and AdS/CFT literature.
} 
can be written in terms of bosonic oscillators $a, a^{\dagger}$ as

$$
S_{-}=a, \quad S_{0}=a^{\dagger} a+s, \quad S_{+}=2 s a^{\dagger}+\left(a^{\dagger}\right)^{2} a .
$$

We will focus on the spin- $\frac{1}{2}$ representation and take $s=1 / 2$ from now on. The generators satisfy the $\mathrm{SL}(2, \mathbb{R})$ algebra

$$
\left[S_{0}, S_{ \pm}\right]= \pm S_{ \pm}, \quad\left[S_{+}, S_{-}\right]=-2 S_{0} .
$$

The local Hilbert space for this spin chain is infinite dimensional. The basis vectors are given by

$$
|n\rangle \equiv \frac{\left(S_{+}\right)^{n}}{n !}|0\rangle, \quad n=1,2, \cdots .
$$

where $|0\rangle$ is the vacuum state defined by

$$
S_{-}|0\rangle=0 .
$$

The action of the generators on the basis is given by

$$
S_{+}|m\rangle=(m+1)|m+1\rangle, \quad S_{-}|m\rangle=m|m-1\rangle, \quad S_{0}|m\rangle=\left(m+\frac{1}{2}\right)|m\rangle .
$$

Similarly, the dual states are defined by

$$
\langle n|=\langle 0| \frac{\left(S_{-}\right)^{n}}{n !}, \quad\langle 0| S_{+}=0
$$

Using the definition of the states and the $\mathrm{SL}(2, \mathbb{R})$ algebra, it is straightforward to show that the basis states are orthonormal

$$
\langle n \mid m\rangle=\delta_{m, n} .
$$

The Hamiltonian takes the local form as in (2.1). The local Hamiltonian density $h_{j, j+1}$ acts on $\mathcal{H}_{j} \otimes \mathcal{H}_{j+1}$ as

$$
\begin{aligned}
h_{j, j+1}\left|m_{j}\right\rangle \otimes\left|m_{j+1}\right\rangle= & \left(h\left(m_{j}\right)+h\left(m_{j+1}\right)\right)\left|m_{j}\right\rangle \otimes\left|m_{j+1}\right\rangle \\
& -\sum_{k=1}^{m_{j}} \frac{1}{k}\left|m_{j}-k\right\rangle \otimes\left|m_{j+1}+k\right\rangle \\
& -\sum_{k=1}^{m_{j+1}} \frac{1}{k}\left|m_{j}+k\right\rangle \otimes\left|m_{j+1}-k\right\rangle .
\end{aligned}
$$

where $h(m)$ is the harmonic sum

$$
h(m)=\sum_{k=1}^{m} \frac{1}{k} .
$$

Like their compact cousins, non-compact spin chains also have many applications in physics. For example, the $\mathrm{SL}(2, \mathbb{C})$ spin chain shows up in the study of Regge limit of QCD [20$22]$. The $\operatorname{SL}(2, \mathbb{R})$ spin chain which we study in this paper first appeared in the study of baryon distribution amplitudes in QCD [18]. Later in integrability in $\mathrm{AdS}_{5} / \mathrm{CFT}_{4}$, this Hamiltonian describes the one-loop dilatation operator of the SL(2) sector. Recently, it also made its appearance in non-equilibrium statistical mechanics [23]. 


\subsection{Coordinate Bethe Ansatz}

Both the compact and non-compact spin chains are integrable and can be solved by Bethe Ansatz. We can use either the coordinate or the algebraic Bethe Ansatz to construct the eigenstates. For our proof below, it is more convenient to use the coordinate Bethe Ansatz. Regarding the spin- $\frac{1}{2}$ chains the method goes back to the works [24-27], whereas for higher spin cases it was worked out in $[28,29]$. In the case of the non-compact chain we can use the results of [28] or those of [29] after analytic continuation to $s=-1 / 2$.

Reference state. The eigenstates are constructed as interacting spin waves over a proper reference state. For compact spin chain, the reference state is chosen to be the ferromagnetic vacuum

$$
|\Omega\rangle=|\uparrow\rangle^{\otimes L}
$$

For the non-compact spin chain, the reference state is chosen to be the Fock vacuum

$$
|\Omega\rangle=|0\rangle^{\otimes L}
$$

The reference states are eigenstates of the Hamiltonians. To obtain other eigenstates, we introduce excitations on top of the vacuum state. A generic eigenstate is characterized by a set of rapidities $\boldsymbol{\lambda}_{N} \equiv\left\{\lambda_{1}, \lambda_{2}, \cdots, \lambda_{N}\right\}$; the corresponding eigenstate will be denoted by $\left|\boldsymbol{\lambda}_{N}\right\rangle$.

Basis vectors. Let us first introduce the basis vectors as

$$
\left|x_{1}, \ldots, x_{N}\right\rangle \sim S_{ \pm}^{\left(x_{1}\right)} S_{ \pm}^{\left(x_{2}\right)} \cdots S_{ \pm}^{\left(x_{N}\right)}|\Omega\rangle
$$

where the $x_{j}$ denote the positions of the sites and $S_{ \pm}^{\left(x_{j}\right)}$ denotes the local spin operator at site $x_{j}$ that creates one excitation. Each $x_{j}$ runs from 1 to $L$. From our convention of reference states, for the compact and non-compact chains the creation operators are $S_{-}^{(x)}$ and $S_{+}^{(x)}$ respectively. Now comes the crucial difference between compact and non-compact spin chains. For the compact spin chain, we can act with $S_{-}^{\left(x_{j}\right)}$ on each site $x_{j}$ only once, thus each site can only hold one excitation. In the contrary, for non-compact spin chain, we can act with any number of $S_{+}^{\left(x_{j}\right)}$ on site $x_{j}$.

In the non-compact case the precise normalization of the basis vectors is given by

$$
\left|x_{1}, \ldots, x_{N}\right\rangle=E_{+}^{\left(x_{1}\right)} E_{+}^{\left(x_{2}\right)} \cdots E_{+}^{\left(x_{N}\right)}|\Omega\rangle
$$

with

$$
E_{+}^{(x)}|m\rangle_{x}=|m+1\rangle_{x} .
$$

The $E_{+}$operators are conjugate to $S_{+}$, and their usage leads to a convenient representation of the coordinate Bethe Ansatz wave functions. See [29] for the detailed discussion of this point. 
The basis states are thus given in the two cases by

$$
\begin{array}{rll}
\text { Compact chain : } & \left|x_{1}, x_{2}, \cdots, x_{N}\right\rangle \quad 1 \leq x_{1}<x_{2} \cdots<x_{N} \leq L, \\
\text { Non-compact chain : } & \left|x_{1}, x_{2}, \cdots, x_{N}\right\rangle \quad 1 \leq x_{1} \leq x_{2} \cdots \leq x_{N} \leq L .
\end{array}
$$

The eigenstate $\left|\boldsymbol{\lambda}_{N}\right\rangle$ is given by a proper linear combination of the basis states

$$
\left|\boldsymbol{\lambda}_{N}\right\rangle=\sum_{\left\{x_{j}\right\}} \chi\left(\boldsymbol{x}_{N}, \boldsymbol{\lambda}_{N}\right)\left|x_{1}, x_{2}, \cdots, x_{N}\right\rangle,
$$

where the range for the summation over $x_{j}$ are given in (2.18).

Bethe wave functions. Now we discuss how to construct the wave function $\chi\left(\boldsymbol{x}_{N}, \boldsymbol{\lambda}_{N}\right)$. It takes the following form:

$$
\chi\left(\boldsymbol{x}_{N}, \boldsymbol{\lambda}_{N}\right)=\sum_{\sigma \in S_{N}} \prod_{j>k} f\left(\lambda_{\sigma_{j}}-\lambda_{\sigma_{k}}\right) \prod_{j=1}^{N} e^{i p_{\sigma_{j}} x_{j}},
$$

where $p_{\sigma_{j}}=p\left(\lambda_{\sigma_{j}}\right)$ is the momentum of the excitation with rapidity $\lambda_{\sigma_{j}} \cdot f(\lambda)$ is certain known function which is related to the $S$-matrix of excitations by

$$
S(\lambda, \mu)=\frac{f(\lambda-\mu)}{f(\mu-\lambda)}
$$

The summation in (2.20) is over all permutations of indices $\{1,2, \cdots, N\}$, which is denoted by $S_{N}$.

Different models are distinguished by the different $p(\lambda)$ and $f(\lambda)$ functions. For the three spin chains under consideration, the two functions are given by

- Compact XXZ chain $(\Delta>1)$

$$
e^{i p(\lambda)}=\frac{\sin (\lambda-i \eta / 2)}{\sin (\lambda+i \eta / 2)}, \quad f(\lambda)=\frac{\sin (\lambda+i \eta)}{\sin (\lambda)}, \quad S(\lambda)=\frac{\sin (\lambda+i \eta)}{\sin (\lambda-i \eta)},
$$

where $\eta$ is related to the anisotropy by $\Delta=\cosh \eta$.

- Compact XXX chain

$$
e^{i p(\lambda)}=\frac{\lambda-i / 2}{\lambda+i / 2}, \quad f(\lambda)=\frac{\lambda+i}{\lambda}, \quad S(\lambda)=\frac{\lambda+i}{\lambda-i} .
$$

- Non-compact chain

$$
e^{i p(\lambda)}=\frac{\lambda-i / 2}{\lambda+i / 2}, \quad f(\lambda)=\frac{\lambda-i}{\lambda}, \quad S(\lambda)=\frac{\lambda-i}{\lambda+i} .
$$

Our sign convention for the rapidity is such that $p^{\prime}(\lambda)>0$ in all cases. 
Bethe equations. Periodicity of the eigenstate implies that the rapidities $\{\boldsymbol{\lambda}\}_{N}$ have to satisfy Bethe equations

$$
e^{i p\left(\lambda_{j}\right) L} \prod_{k \neq j} S\left(\lambda_{j}-\lambda_{k}\right)=1 .
$$

The rapidities can be found by solving Bethe equations. After finding the rapidities, the eigenvalue of the Hamiltonian is given by the total energy of the system

$$
H\left|\left\{\boldsymbol{\lambda}_{N}\right\}\right\rangle=E_{N}\left(\{\boldsymbol{\lambda}\}_{N}\right)\left|\left\{\boldsymbol{\lambda}_{N}\right\}\right\rangle, \quad E_{N}\left(\{\boldsymbol{\lambda}\}_{N}\right)=\sum_{j=1}^{N} e\left(\lambda_{j}\right) .
$$

For the XXX spin chains (both compact and non-compact) the function $e(\lambda)$ is given by

$$
e(\lambda)=-\frac{2}{\lambda^{2}+\frac{1}{4}} .
$$

For the XXZ spin chain, the function is given by

$$
e(\lambda)=\frac{4 \sinh ^{2} \eta}{\cos (2 \lambda)-\cosh \eta} .
$$

Some notations. For future use let us introduce the variables

$$
l_{j}=e^{i p\left(\lambda_{j}\right)} .
$$

It follows from the concrete formulae (2.23)-(2.22) that $f\left(\lambda_{j}-\lambda_{k}\right)$ is a rational function of $l_{j}, l_{k}$. With some abuse of notation we will write it as $f\left(l_{j}, l_{k}\right)$. We can thus regard the Bethe wave function as a rational function of the $l$-variables:

$$
\chi\left(\boldsymbol{x}_{N}, \boldsymbol{\lambda}_{N}\right)=\sum_{\sigma \in S_{N}} \prod_{j>k} f\left(l_{\sigma_{j}}, l_{\sigma_{k}}\right) \prod_{j=1}^{N}\left(l_{\sigma_{j}}\right)^{x_{j}} .
$$

This representation will play an important role in the overlap computations. The Bethe equations are rewritten as

$$
a_{j}=\prod_{k \neq j} \frac{f\left(l_{k}, l_{j}\right)}{f\left(l_{j}, l_{k}\right)}
$$

where we introduced the $a$-variables as

$$
a_{j}=l_{j}^{L}=e^{i p_{j} L} .
$$

\section{$3 \quad$ Integrable boundary states}

In this section, we discuss integrable boundary states for integrable spin chains. We first review the proposal of [10] for characterizing integrable boundary states for general spin chains. Although the proposal was motivated for compact spin chains, it is straightforward to generalize it to the non-compact cases. On the other hand, some techniques for the explicit constructions of the boundary states rely on the rotation trick and do not allow for an immediate generalization to the non-compact case. 
After the general discussion, we focus on explicit examples for the compact and noncompact spin chains. The discussion for the compact cases mainly just reviews the known results. The results on integrable boundary states of non-compact spin chains are new. Finally we give the explicit formula for the exact overlap between a Bethe state and the integrable state, which will be proven in later sections.

\subsection{General discussion}

We review the definition of integrable boundary states according to [10], which is inspired from the definition of boundary states in quantum field theories [14].

Integrable models possess a family of conserved charges that are in involution with each other:

$$
\left[Q_{\alpha}, Q_{\beta}\right]=0 .
$$

In local spin chains these charges are also local, which means they can be written in the form

$$
Q_{\alpha}=\sum_{x=1}^{L} q_{\alpha}(x) .
$$

where $q_{\alpha}(x)$ is a local operator whose range can be chosen to be $\alpha$. In other words it only acts on sites $x, x+1, \ldots, x+\alpha-1$. The Hamiltonian of the spin chain is one of the conserved charges, and usually we choose $H \sim Q_{2}$.

Let $\Pi$ be the space parity operator which acts on the basis vector $\left|i_{1}, i_{2}, \cdots, i_{L}\right\rangle$ as

$$
\Pi\left|i_{1}, i_{2}, \cdots, i_{L}\right\rangle=\left|i_{L}, i_{L-1}, \cdots, i_{1}\right\rangle \text {. }
$$

The charges can be chosen in such a way that they have fixed parity under space reflection

$$
\Pi Q_{\alpha} \Pi=(-1)^{\alpha} Q_{\alpha}, \quad \alpha \geq 2 .
$$

Integrable boundary states $|\Psi\rangle$ are defined as the elements of the Hilbert space satisfying the condition

$$
Q_{2 k+1}|\Psi\rangle=0, \quad k=1,2, \ldots
$$

A perhaps more natural integrability condition can be given using the transfer matrix (TM), which generates the set of conserved charges. Such a TM can usually be constructed systematically in the algebraic Bethe Ansatz. In the following we briefly review this construction.

In the local integrable spin chains related to the Lie-group $G$ there is a rapidity dependent $\operatorname{TM} t^{\Lambda}(u)$ for all representation $\Lambda$ of $G$, such that for all $\Lambda, \Lambda^{\prime}$ :

$$
\left[t^{\Lambda}(u), t^{\Lambda^{\prime}}\left(u^{\prime}\right)\right]=0
$$

These transfer matrices are constructed using Lax operators as

$$
t^{\Lambda}(u)=\operatorname{Tr}_{a} T_{a}^{\Lambda}(u), \quad T_{a}(u)=\prod_{k=1}^{L} L_{a k}^{\Lambda}(u) .
$$


Here $L_{a, k}^{\Lambda}$ are the so-called Lax operators, $k$ is the index of the local Hilbert spaces, and $a$ stands for an auxiliary space, carrying the representation $\Lambda$ of the group $G$.

Typically there are two distinguished transfer matrices, corresponding to the cases below:

- $\Lambda$ is the defining representation of the group $G$. The corresponding TM will be called "fundamental" and it will be denoted as $\tau(u)$.

- $\Lambda$ is the representation of the physical spaces. The corresponding TM will be called "physical" and it will be denoted as $t^{0}(u)$.

In our cases $G=\mathrm{SU}(2)$. In the compact XXX case the physical spaces carry the defining representation, therefore the two TM's mentioned above coincide. However, in the higher spin cases and in the non-compact chain they are different.

Typically the physical TM is used to generated the local conserved charges. Expanding it in a power series we define (see for example $[30]$ and $[19,21]$ for the non-compact cases)

$$
t^{0}(u)=U \exp \left(\sum_{n=1}^{\infty} \beta_{n} \frac{u^{n}}{n !} Q_{n+1}\right),
$$

where $\beta_{n}$ are chosen to make the charges $Q_{n+1}$ Hermitian. $U=t^{0}(0)$ is the the translation or shift operator.

It follows from this expansion that the integrability condition for the boundary state can be written as

$$
t^{0}(u)|\Psi\rangle=\Pi t^{0}(u) \Pi|\Psi\rangle .
$$

Several important remarks are in order.

First, this condition is somewhat stronger than (3.5), because it also implies

$$
U^{2}|\Psi\rangle=|\Psi\rangle,
$$

which does not follow from (3.5). Although it has not yet been proven rigorously that (3.5) implies (3.9), in interacting models there is no known case where the two-site invariance (3.10) is not satisfied.

We can also require an integrability condition using the defining TM:

$$
\tau(u)|\Psi\rangle=\Pi \tau(u) \Pi|\Psi\rangle .
$$

The equivalence of (3.11) and (3.9) is not guaranteed. Typically the different transfer matrices are algebraically dependent, which is established through the so-called fusion relations (also known as the Hirota equation). In the case of $G=\mathrm{SU}(2)$ these fusion relations guarantee that (3.11) and (3.9) are equivalent, but for higher rank groups it is possible that the integrability conditions with TM's corresponding to different representations have a different form [31].

We now give the explicit construction of the fundamental transfer matrix with the $\mathrm{SU}(2)$-symmetry, both in the compact and non-compact ones. The Lax operator at each site- $j$ is given by

$$
L_{a j}(u)=u+i\left(\vec{\sigma}_{a} \cdot \vec{S}_{j}\right)=u+i\left(\sigma_{a}^{z} S_{j}^{z}+\sigma_{a}^{-} S_{j}^{+}+\sigma_{a}^{+} S_{j}^{-}\right),
$$


where it is understood that $S_{ \pm}=S_{x} \pm i S_{y}$, and for the compact spin chain $S^{\alpha}=\frac{1}{2} \sigma^{\alpha}$, whereas for the non-compact spin chain the $S^{z}, S^{ \pm}$operators are given by (2.4).

This TM satisfies a crossing relation. The Pauli matrices satisfy the relation $\sigma^{y} \sigma^{a} \sigma^{y}=$ $-\left(\sigma^{a}\right)^{\mathrm{T}}$ with $a=x, y, z$, where the superscript ${ }^{\mathrm{T}}$ denotes transposition. This implies

$$
\sigma_{a}^{y} L_{a j}(u) \sigma_{a}^{y}=-L_{a j}^{\mathrm{T}_{a}}(-u)
$$

For the TM this means

$$
\tau(-u)=\Pi \tau(u) \Pi .
$$

The integrability condition is therefore equivalent to

$$
\tau(u)|\Psi\rangle=\tau(-u)|\Psi\rangle .
$$

We stress that this is not a generic feature of integrable models, and it is only valid for the defining representation of the $\mathrm{SU}(2)$-related models, and only with our specific choice for the additive and multiplicative normalization of the local Lax operators.

In the non-compact case the integrability conditions have not yet been discussed before. We take (3.11) (or the equivalent conditon (3.15)) as the fundamental definition of integrability for the non-compact chain. Now we show that this ensures the pair property for the overlaps, and thus the original condition (3.5) and also (3.9) will be satisfied.

It can be derived using the Algebraic Bethe Ansatz [30], that the eigenvalue of the fundamental transfer matrix on the Bethe state given by (2.20) is

$$
\tau(u)=(u+i / 2)^{L} \prod_{j=1}^{N} f\left(\lambda_{j}-u\right)+(u-i / 2)^{L} \prod_{j=1}^{N} f\left(u-\lambda_{j}\right) .
$$

Here we used the same notation $\tau(u)$ also for the eigenvalue. This formula holds both in the compact XXX case and the non-compact chain, with the $f$-functions given by $(2.23)$ and (2.24), respectively. It follows directly from the integrability condition (3.15) that the overlaps can be non-zero only when the corresponding eigenvalues satisfy $\tau(u)=\tau(-u)$. This immediately leads to the requirement that the set $\boldsymbol{\lambda}_{N}$ be parity symmetric, both in the compact and non-compact cases.

\subsection{The compact chains}

In the literature two main classes of integrable states have been considered. The first class is the two-site states which are defined as

$$
|\Psi\rangle=\otimes_{j=1}^{L / 2}|\psi\rangle, \quad|\psi\rangle \in \mathbb{C}^{2} \otimes \mathbb{C}^{2} .
$$

It was shown in [10] that in the XXX and XXZ models every two-site state is integrable. Furthermore they correspond to integrable $K$-matrices through

$$
\psi_{a b}=(K(\sigma) C)_{a}^{b}
$$

where $C$ is constant matrix describing the so-called crossing transformation and $\sigma$ is a special value for the rapidity parameter (for details see [10]). The $K$-matrix describes an 
integrable boundary condition, and it satisfies the standard Boundary Yang-Baxter (BYB) relation

$$
K_{2}(v) R_{21}(u+v) K_{1}(u) R_{12}(v-u)=R_{21}(v-u) K_{1}(u) R_{12}(u+v) K_{2}(v),
$$

where $R(u)$ is the so-called $R$-matrix in the fundamental representation, see [10].

The physical meaning of the correspondence (3.18) is that an integrable boundary in space (described by the $K$-matrix) is transformed into an integrable boundary in time (described by the boundary state). This is the generalization of the same picture in integrable QFT, first developed by Ghoshal and Zamolodchikov [14].

Another class of states is given by integrable matrix product states (MPS) defined as

$$
|\Psi\rangle=\sum_{j_{1}, \ldots, j_{L}=1}^{2} \operatorname{Tr}_{A}\left[\omega_{j_{L}} \ldots \omega_{j_{2}} \omega_{j_{1}}\right]\left|j_{L}, \ldots, j_{2}, j_{1}\right\rangle
$$

Here $\omega_{j}, j=1,2$ are matrices acting on one more auxiliary space denoted by $A$. The study of such integrable MPS was initiated in the works $[4,5]$, and later it was shown in [13] that these states are also described by solutions of the BYBE, although the corresponding $K$-matrices have an inner degree of freedom. The work [13] also treated two-site invariant MPS, and the two-site states above can be considered as MPS with "trivial", one dimensional auxiliary space.

It was argued in [11] that in the $\mathrm{SU}(2)$-symmetric chains all integrable MPS are obtained by the action of transfer matrices on two-site states. This is not true in spin chains with higher rank symmetries: the works $[12,13]$ treated a number of "indecomposable" MPS's.

We note that in the higher rank cases there are two main types of integrable boundary conditions, described by the original and the twisted BYB relations. The integrable initial states are always related to the twisted case [13]. However, in the $\mathrm{SU}(2)$ and $\mathrm{SO}(N)$ related models the two types of boundary conditions are equivalent, which can be shown by a crossing relation, see [13] for a detailed discussion on this issue. Here we do not treat this distinction and only refer to the original BYB (3.19).

\subsection{The non-compact chain}

Much less is known about integrable boundary states for non-compact spin chains compared to the compact case. Here we present the first example which satisfy the integrability conditions. It appears in the context of AdS/CFT [1] and an exact overlap formula has been proposed. This integrable boundary state can be seen as a counterpart of the generalized Néel state in the compact case [32].

A generalized Néel state. To introduce the integrable boundary state, it is more convenient to write the basis vectors of the Hilbert space as

$$
\left|n_{1}, n_{2}, \cdots, n_{L}\right\rangle \equiv\left|n_{1}\right\rangle \otimes\left|n_{2}\right\rangle \otimes \cdots \otimes\left|n_{L}\right\rangle
$$


where $n_{j}$ denotes the number of excitations at the site- $j$; to be more precise

$$
\left|n_{j}\right\rangle=\frac{\left(S_{+}\right)^{n_{j}}}{n_{j} !}\left|0_{j}\right\rangle
$$

Assuming that $L$ is even, we define a family of states which depend on a free parameter $\kappa$ :

$$
\left|\mathrm{Néel}_{\kappa}\right\rangle=\sum_{\left\{n_{i}\right\}}\left(\kappa^{N_{\text {odd }}}+\kappa^{N_{\text {even }}}\right)\left|n_{1}, n_{2}, \cdots, n_{L}\right\rangle,
$$

where the summation for each $n_{j}$ runs over all non-negative integers. $N_{\text {odd }}$ and $N_{\text {even }}$ are the total number of excitations on odd and even sites

$$
N_{\text {odd }}=n_{1}+n_{3}+\cdots+n_{L-1}, \quad N_{\text {even }}=n_{2}+n_{4}+\cdots+n_{L} .
$$

There are two special cases for this generalized Néel state. This first one is $\kappa=1$, where $\mid$ Néel $\left._{\kappa=1}\right\rangle$ is simply the sum over all basis vectors of the Hilbert space. We will denote this state by $\left|X_{F}\right\rangle$ in what follows; it is a one-site invariant ferromagnetic state.

The second special case is $\kappa=0$. It follows from (3.23) that the non-vanishing contributions at $\kappa=0$ are given by $N_{\text {odd }}=0$ or $N_{\text {even }}=0$. The state $\mid$ Néel $\left._{0}\right\rangle$ takes the form

$$
\left|\mathrm{Néel}_{0}\right\rangle=\sum_{\substack{|j-k| \\ \text { even }}}\left|\circ \cdots \circ \bullet_{j} \circ \cdots \circ \bullet_{k} \circ \cdots\right\rangle,
$$

where the black dots stand for possible positions of excitations, and the sum is taken over all possible distributions under the restriction that the distances between the black dots have to be even. For example, for $L=4$, we have the following state

$$
\begin{aligned}
\left.\mid \text { Néel }_{0}\right\rangle= & |\circ \circ \circ \circ\rangle+|\bullet \circ \circ \circ\rangle+|\circ \bullet \circ \circ\rangle+|\circ \circ \bullet \circ\rangle+|\circ \circ \circ \bullet\rangle \\
& +|\circ \bullet \circ \bullet\rangle+|\bullet \circ \bullet \circ\rangle
\end{aligned}
$$

It is easy to see that the number of black dots cannot be larger than $L / 2$. The precise normalization for this notation is given by

$$
|\circ\rangle \equiv|0\rangle, \quad|\bullet\rangle \equiv \sum_{n=1}^{\infty}|n\rangle .
$$

Noticing that

$$
e^{S_{+}}|0\rangle=|0\rangle+\sum_{n=1}^{\infty} \frac{\left(S_{+}\right)^{n}}{n !}|0\rangle=|0\rangle+\sum_{n=1}^{\infty}|n\rangle=|0\rangle+|\bullet\rangle,
$$

it is easy to see that $\left|\mathrm{Née}_{\kappa}\right\rangle$ can be written as

$$
\left|\mathrm{Néel}_{\kappa}\right\rangle=\left(e^{\kappa S_{+}}|0\rangle \otimes e^{S_{+}}|0\rangle\right)^{L / 2}+\left(e^{S_{+}}|0\rangle \otimes e^{\kappa S_{+}}|0\rangle\right)^{L / 2} .
$$

Alternatively we can write

$$
\left|\mathrm{Néel}_{\kappa}\right\rangle=e^{\kappa \mathcal{S}_{+}}\left|\Psi_{1-\kappa}\right\rangle,
$$


where $\mathcal{S}_{+}=S_{+}^{(1)}+S_{+}^{(2)}+\cdots S_{+}^{(L)}$ is the $\mathrm{SL}(2, \mathbb{R})$ generator for the full spin chain. The state $\left|\Psi_{\alpha}\right\rangle$ is defined by

$$
\left|\Psi_{\alpha}\right\rangle=(|0\rangle \otimes|\alpha\rangle)^{L / 2}+(|\alpha\rangle \otimes|0\rangle)^{L / 2},
$$

where $|\alpha\rangle$ is the coherent state $|\alpha\rangle=e^{\alpha S_{+}}|0\rangle$. An on-shell Bethe state is the highest weight state of $\mathrm{SL}(2, \mathbb{R})$ and hence

$$
\mathcal{S}_{-}\left|\boldsymbol{\lambda}_{N}\right\rangle=0
$$

Therefore we have

$$
\left\langle\mathrm{Néel}_{\kappa} \mid \boldsymbol{\lambda}_{N}\right\rangle=\left\langle\Psi_{1-\kappa}\left|e^{\kappa \mathcal{S}_{-}}\right| \boldsymbol{\lambda}_{N}\right\rangle=\left\langle\Psi_{1-\kappa} \mid \boldsymbol{\lambda}_{N}\right\rangle
$$

From the definition of $\left|\Psi_{\alpha}\right\rangle$, it is easy to see that

$$
\left\langle\Psi_{\alpha} \mid \boldsymbol{\lambda}_{N}\right\rangle=\alpha^{N}\left\langle\mathrm{Néel}_{0} \mid \boldsymbol{\lambda}_{N}\right\rangle
$$

Combing this equation with (3.33), we arrive at the following relation:

$$
\left\langle\operatorname{Néel}_{\kappa} \mid \boldsymbol{\lambda}_{N}\right\rangle=(1-\kappa)^{N}\left\langle\operatorname{Néel}_{0} \mid \boldsymbol{\lambda}_{N}\right\rangle,
$$

where $N$ is the number of rapidities of $\left|\boldsymbol{\lambda}_{N}\right\rangle$.

Now we prove that $\left|\Psi_{\alpha}\right\rangle$ is indeed integrable by the criteria given in section 3.1, namely the condition (3.11) holds for it. The strategy for the proof of integrability was developed in [13], a closely related method already appeared in [33]. The idea is to write both sides of (3.11) as a MPS, and to find a similarity transformation that connects the matrices involved. This similarity transformation can be identified with the integrable $K$-matrix [13].

First of all, it is clear that

$$
\Pi\left|\Psi_{\alpha}\right\rangle=\left|\Psi_{\alpha}\right\rangle
$$

To proceed, it is useful to compute the action of the Lax operator at each site. We have

$$
L_{a j}(u)|0\rangle_{j}=\left(\begin{array}{cc}
u+\frac{i}{2} & 0 \\
0 & u-\frac{i}{2}
\end{array}\right)|0\rangle_{j}+\left(\begin{array}{cc}
0 & 0 \\
i & 0
\end{array}\right) S_{+}^{(j)}|0\rangle_{j}
$$

and

$$
L_{a j}(u)|\alpha\rangle_{j}=\left(\begin{array}{cc}
u+\frac{i}{2} & i \alpha \\
0 & u-\frac{i}{2}
\end{array}\right)|\alpha\rangle_{j}+\left(\begin{array}{cc}
i \alpha & i \alpha^{2} \\
i & -i \alpha
\end{array}\right) S_{+}^{(j)}|\alpha\rangle_{j},
$$

where we have used (A.3) which is derived in the appendix. Taking direct product of two sites, we have

$$
\begin{aligned}
L_{a, j}(u) L_{a, j+1}(u)|0, \alpha\rangle_{j, j+1} & \left.=\sum_{i=1}^{4} A_{i}|i\rangle\right\rangle_{j, j+1}, \\
L_{a, j}(u) L_{a, j+1}(u)|\alpha, 0\rangle_{j, j+1} & \left.=\sum_{i=1}^{4} \tilde{A}_{i}|\tilde{i}\rangle\right\rangle_{j, j+1} .
\end{aligned}
$$


The states are given by

$$
\begin{array}{rlrl}
|1\rangle\rangle_{j, j+1} & =|0\rangle_{j} \otimes|\alpha\rangle_{j+1}, & & |\tilde{1}\rangle\rangle_{j, j+1}=|\alpha\rangle_{j} \otimes|0\rangle_{j+1}, \\
|2\rangle\rangle_{j, j+1}=S_{+}^{(j)}|0\rangle_{j} \otimes|\alpha\rangle_{j+1}, & & |\tilde{2}\rangle\rangle_{j, j+1}=|\alpha\rangle_{j} \otimes S_{+}^{(j+1)}|0\rangle_{j+1}, \\
|3\rangle\rangle_{j, j+1}=|0\rangle_{j} \otimes S_{+}^{(j+1)}|\alpha\rangle_{j+1}, & & |\tilde{3}\rangle\rangle_{j, j+1}=S_{+}^{(j)}|\alpha\rangle_{j} \otimes|0\rangle_{j+1}, \\
|4\rangle\rangle_{j, j+1}=S_{+}^{(j)}|0\rangle_{j} \otimes S_{+}^{(j+1)}|\alpha\rangle_{j+1}, & & |\tilde{4}\rangle\rangle_{j, j+1}=S_{+}^{(j)}|\alpha\rangle_{j} \otimes S_{+}^{(j+1)}|0\rangle_{j+1} .
\end{array}
$$

The matrices $A_{i}$ and $\tilde{A}_{i}$ are given by

$$
\begin{array}{ll}
A_{1}=\left(\begin{array}{cc}
(u+i / 2)^{2} & i \alpha(u+i / 2) \\
0 & (u-i / 2)^{2}
\end{array}\right), & \tilde{A}_{1}=\left(\begin{array}{cc}
(u+i / 2)^{2} & i \alpha(u-i / 2) \\
0 & (u-i / 2)^{2}
\end{array}\right), \\
A_{2}=\left(\begin{array}{cc}
0 & 0 \\
i(u+i / 2) & -\alpha
\end{array}\right), & \tilde{A}_{2}=\left(\begin{array}{cc}
-\alpha & 0 \\
i(u-i / 2) & 0
\end{array}\right) \\
A_{3}=\left(\begin{array}{cc}
i \alpha(u+i / 2) & i \alpha^{2}(u+i / 2) \\
i(u-i / 2) & -i \alpha(u-i / 2)
\end{array}\right), & \tilde{A}_{3}=\left(\begin{array}{cc}
i \alpha(u+i / 2) & i \alpha^{2}(u-i / 2) \\
i(u+i / 2) & -i \alpha(u-i / 2)
\end{array}\right) \\
A_{4}=\left(\begin{array}{cc}
0 & 0 \\
-\alpha-\alpha^{2}
\end{array}\right), & \tilde{A}_{4}=\left(\begin{array}{cc}
-\alpha^{2} & 0 \\
\alpha & 0
\end{array}\right) .
\end{array}
$$

A crucial observation for our proof is that $A_{i}$ and $\tilde{A}_{i}$ are related by

$$
\tilde{K}(u) A_{i} \tilde{K}(u)^{-1}=\tilde{A}_{i}^{\mathrm{T}}, \quad i=1,2,3,4,
$$

with the matrix $\tilde{K}(u)$ given by

$$
\tilde{K}(u)=\left(\begin{array}{cc}
2 u & (u+i / 2) \alpha \\
(u-i / 2) \alpha & 0
\end{array}\right) .
$$

It was shown in [13] that such intertwiners can be interpreted as integrable $K$-matrices. In fact, defining

$$
K(u)=\tilde{K}(u) \sigma^{y}
$$

we obtain a solution to the BYB equations (3.19). The presence of the crossing matrix $\sigma^{y}$ is a feature of the $\mathrm{SU}(2)$-related models, see the discussion above.

Using (3.39) we can write down the action of the transfer matrix on $|\Psi\rangle$ as

$$
\begin{aligned}
\tau(u)\left|\Psi_{\alpha}\right\rangle & =\operatorname{Tr}(L(u)|0\rangle \otimes L(u)|\alpha\rangle)^{L / 2}+\operatorname{Tr}(L(u)|\alpha\rangle \otimes L(u)|0\rangle)^{L / 2} \\
& =\operatorname{Tr}\left[A_{i_{1}} A_{i_{2}} \cdots A_{i_{L / 2}}\right]\left|i_{1}, i_{2}, \cdots, i_{L / 2}\right\rangle+\operatorname{Tr}\left[\tilde{A}_{i_{1}} \tilde{A}_{i_{2}} \cdots \tilde{A}_{i_{L / 2}}\right]\left|\tilde{i}_{1}, \tilde{i}_{2}, \cdots, \tilde{i}_{L / 2}\right\rangle,
\end{aligned}
$$

where repeated indices are summed over from 1 to 4 and the trace is taken over the auxiliary space. The states are defined by

$$
\begin{aligned}
& \left.\left.\left.\left|i_{1}, i_{2}, \cdots, i_{L / 2}\right\rangle=\left|i_{1}\right\rangle\right\rangle \otimes\left|i_{2}\right\rangle\right\rangle \otimes \cdots \otimes\left|i_{L / 2}\right\rangle\right\rangle, \\
& \left.\left.\left.\left|\tilde{i}_{1}, \tilde{i}_{2}, \cdots, \tilde{i}_{L / 2}\right\rangle=\left|\tilde{i}_{1}\right\rangle\right\rangle \otimes\left|\tilde{i}_{2}\right\rangle\right\rangle \otimes \cdots \otimes\left|\tilde{i}_{L / 2}\right\rangle\right\rangle .
\end{aligned}
$$


Acting the reflection operator, we obtain

$$
\begin{aligned}
\Pi \tau(u)\left|\Psi_{\alpha}\right\rangle & =\operatorname{Tr}\left[A_{i_{1}} \cdots A_{i_{L / 2}}\right] \Pi\left|i_{1}, \cdots, i_{L / 2}\right\rangle+\operatorname{Tr}\left[\tilde{A}_{i_{1}} \cdots \tilde{A}_{i_{L / 2}}\right] \Pi\left|\tilde{i}_{1}, \cdots, \tilde{i}_{L / 2}\right\rangle \\
& =\operatorname{Tr}\left[A_{i_{1}} \cdots A_{i_{L / 2}}\right]\left|\tilde{i}_{L / 2}, \cdots, \tilde{i}_{1}\right\rangle+\operatorname{Tr}\left[\tilde{A}_{i_{1}} \cdots \tilde{A}_{i_{L / 2}}\right]\left|i_{L / 2}, \cdots, i_{1}\right\rangle .
\end{aligned}
$$

Now using the relation (3.42) we can show easily

$$
\begin{aligned}
\operatorname{Tr}\left[\tilde{A}_{i_{1}} \cdots \tilde{A}_{i_{L / 2}}\right] & =\operatorname{Tr}\left[A_{i_{L / 2}} \cdots A_{i_{1}}\right], \\
\operatorname{Tr}\left[A_{i_{1}} \cdots A_{i_{L / 2}}\right] & =\operatorname{Tr}\left[\tilde{A}_{i_{L / 2}} \cdots \tilde{A}_{i_{1}}\right] .
\end{aligned}
$$

Plugging into the second line of (3.47), we find

$$
\Pi \tau(u)\left|\Psi_{\alpha}\right\rangle=\Pi \tau(u) \Pi\left|\Psi_{\alpha}\right\rangle=\tau(u)\left|\Psi_{\alpha}\right\rangle
$$

which demonstrates that the state $\left|\Psi_{\alpha}\right\rangle$ is an integrable boundary state.

\subsection{Exact overlap formulae}

The integrability condition for the boundary state $|\Psi\rangle$ leads to a number of non-trivial consequences which we discuss below.

Paired Bethe roots. It was first argued in [10], that the condition (3.9) imposes a strict selection rule for the overlaps between $|\Psi\rangle$ and on-shell Bethe states $\left|\boldsymbol{\lambda}_{N}\right\rangle$. Namely, the overlap

$$
\left\langle\Psi \mid \boldsymbol{\lambda}_{N}\right\rangle
$$

is non-zero only if the set of the Bethe roots is parity symmetric. In the case of an even number of particles this means that they come in pairs:

$$
\left\{\boldsymbol{\lambda}_{N}\right\}=\left\{\lambda_{1},-\lambda_{1}, \cdots, \lambda_{N / 2},-\lambda_{N / 2}\right\}
$$

which will also be denoted as

$$
\left\{\boldsymbol{\lambda}_{N}\right\}=\left\{\boldsymbol{\lambda}_{N / 2}^{+},-\boldsymbol{\lambda}_{N / 2}^{+}\right\}
$$

Here $\left\{\boldsymbol{\lambda}_{N / 2}^{+}\right\}$denotes the positive Bethe roots. ${ }^{2}$ When the number of particles is odd, we have

$$
\left\{\boldsymbol{\lambda}_{N}\right\}=\left\{\lambda_{1},-\lambda_{1}, \cdots, \lambda_{(N-1) / 2},-\lambda_{(N-1) / 2}, 0\right\} .
$$

In this work we only consider overlaps with Bethe states with even numbers of particles. The cases with odd number of Bethe roots can be treated similarly. For earlier studies with an odd number of particles see [12, 15, 34].

\footnotetext{
${ }^{2}$ In principle, it does not matter which root among the pair we call 'positive'. As a convention, we can choose the one with positive real part as the positive Bethe root.
} 
Factorized Gaudin norm. It is well-known that the norm of the on-shell Bethe state constructed in (2.30) can be expressed as [17]

$$
\left\langle\boldsymbol{\lambda}_{N} \mid \boldsymbol{\lambda}_{N}\right\rangle=\prod_{j=1}^{N} \frac{1}{p^{\prime}\left(\lambda_{j}\right)} \prod_{j<k}^{N} f\left(\lambda_{j}-\lambda_{k}\right) f\left(\lambda_{k}-\lambda_{j}\right) \times \operatorname{det} G
$$

where $G$ is an $N \times N$ matrix known as the Gaudin matrix whose elements are

$$
G_{j k}=\delta_{j k}\left[p^{\prime}\left(\lambda_{j}\right) L+\sum_{l=1}^{N} \varphi\left(\lambda_{j}-\lambda_{l}\right)\right]-\varphi\left(\lambda_{j}-\lambda_{k}\right) .
$$

The function $\varphi(\lambda)$ is defined as

$$
\varphi(\lambda)=-i \frac{d}{d \lambda} \log S(\lambda)
$$

The norm of an on-shell Bethe state whose rapidities are paired as in (3.51) factorizes further. For such symmetric states the Gaudin matrix has a block structure and the determinant can be factorized as

$$
\operatorname{det} G=\operatorname{det} G^{+} \operatorname{det} G^{-},
$$

where $G^{ \pm}$are $\frac{N}{2} \times \frac{N}{2}$ matrices with matrix elements

$$
G_{j k}^{ \pm}=\delta_{j k}\left[p^{\prime}\left(\lambda_{j}^{+}\right) L+\sum_{l=1}^{N / 2} \varphi^{+}\left(\lambda_{j}^{+}, \lambda_{l}^{+}\right)\right]-\varphi^{ \pm}\left(\lambda_{j}^{+}, \lambda_{k}^{+}\right)
$$

with

$$
\varphi^{ \pm}(\lambda, \mu)=\varphi(\lambda-\mu) \pm \varphi(\lambda+\mu) .
$$

The norm is then written as

$$
\left\langle\boldsymbol{\lambda}_{N} \mid \boldsymbol{\lambda}_{N}\right\rangle=\prod_{j=1}^{N / 2} \frac{f\left(2 \lambda_{j}^{+}\right) f\left(-2 \lambda_{j}^{+}\right)}{\left(p^{\prime}\left(\lambda_{j}^{+}\right)\right)^{2}} \prod_{1 \leq j<k \leq N / 2}\left[\bar{f}\left(\lambda_{j}^{+}, \lambda_{k}^{+}\right)\right]^{2} \times \operatorname{det} G^{+} \operatorname{det} G^{-},
$$

where we defined

$$
\bar{f}(\lambda, \mu)=f(\lambda-\mu) f(\lambda+\mu) f(-\lambda-\mu) f(-\lambda+\mu) .
$$

Exact overlap formulae. The most important property is that the non-zero overlaps between many integrable boundary states and on-shell Bethe states take a remarkably simple form:

$$
\frac{\left|\left\langle\Psi \mid \boldsymbol{\lambda}_{N}\right\rangle\right|^{2}}{\left\langle\boldsymbol{\lambda}_{N} \mid \boldsymbol{\lambda}_{N}\right\rangle}=\prod_{j=1}^{N / 2} u\left(\lambda_{j}^{+}\right) \times \frac{\operatorname{det} G^{+}}{\operatorname{det} G^{-}}
$$

Here $u(\lambda)$ is the so-called one particle overlap function, which depends on the initial state, and $G^{ \pm}$are the same matrices that appeared in the factorized Gaudin norm. Below we will prove this overlap formula in a number of cases. 
If the integrable boundary state is a simple product state, then all known cases involve only a single product as in (3.62). However, for other states such as the integrable MPS, the pre-factor in front of the ratio of determinants can take more complicated forms. For more details, see the discussions in [12]. We put forward that our present method allows for a rigorous proof only in those cases when the overlap involves only a single product.

The simple form for the exact overlap formula (3.62) seems to hold for both the compact and non-compact spin chains. In the case of the compact chain the one-particle overlap function $u(\lambda)$ can be determined by a "rotation trick" $[10,11]$. The idea is to relate the quantum system to a 2 dimensional classical lattice model, and to build partition functions that are afterwards evaluated using the so called Quantum Transfer Matrix in the "rotated channel", after rotating the lattice by $90^{\circ}$. For non-compact spin chains, the local Hilbert space at each site is infinite dimensional and the rotation trick cannot be applied in a straightforward way. Therefore a new method is called for. Below we develop such a method for proving the exact overlap formula of the non-compact spin chain.

\section{Exact overlap formulae - General strategy}

In this section we explain the general strategy of our method. We postpone the concrete computations for different integrable boundary states to section 5 .

The method is most easily demonstrated on the compact XXX and XXZ chains, with the initial state being

$$
|\Psi\rangle=\left|X_{F}\right\rangle \equiv \otimes_{j=1}^{L}\left(\begin{array}{l}
1 \\
1
\end{array}\right) .
$$

The overlap of a given Bethe state with this state is particularly simple, because each spin configuration has the same weight in the overlap. The result is thus simply the sum over the wave function coefficients.

Regarding the Bethe states as given by (2.30), the un-normalized overlaps are

$$
\left\langle X_{F} \mid \boldsymbol{\lambda}_{N}\right\rangle=\sum_{\sigma \in S_{N}} \prod_{j>k} f\left(l_{\sigma_{j}}, l_{\sigma_{k}}\right) \sum_{0 \leq x_{1}<\cdots<x_{N} \leq L-1} \prod_{j=1}^{N} l_{\sigma_{j}}^{x_{j}} .
$$

Such an overlap is a rational function of the set $\left\{l_{1}, \ldots, l_{N}\right\}$. For this set of variables we will also use the notation $\boldsymbol{l}_{N}$.

We want to evaluate this rational function for the $\boldsymbol{l}_{N}$ which satisfy the Bethe equations (2.31). These equations depend on $L$, therefore the first natural question is: how do the overlaps depend on the length of the spin chain $L$ ?

The scalar products (4.2) carry a formal dependence on $L$, which is hidden in the summation limits. It is our goal to make this dependence more explicit. We will see that the summations can be performed using algebraic manipulations, such that eventually (4.2) will be expressed as rational functions of two sets of variables $\boldsymbol{l}_{N}$ and $\boldsymbol{a}_{N}=\left\{a_{1}, \ldots, a_{N}\right\}$, where the $a$-variables were introduced in (2.32). We will see that there will be no further $L$ dependence. It will be this rational function where we can "substitute the Bethe equations" such that the on-shell values of the overlaps can be obtained.

In order to explain the method we first consider the simplest examples. 


\subsection{One-particle states}

In this case the overlap is given by the simple sum

$$
\left\langle X_{F} \mid \lambda_{1}\right\rangle=\sum_{j=0}^{L-1} l_{1}^{j}
$$

This sum can be computed readily

$$
\left\langle X_{F} \mid \lambda_{1}\right\rangle=\left\{\begin{array}{ll}
L & \text { if } l_{1}=1 \\
\frac{a_{1}-1}{l_{1}-1} & \text { if } l_{1} \neq 1
\end{array} .\right.
$$

Here we already used the new auxiliary variable $a_{1}=l_{1}^{L}$.

The above formulae refer to the off-shell case: they are valid for arbitrary $l_{1}$. Let us now investigate the on-shell case. In the one-particle case the Bethe equation is simply

$$
a_{1}=\left(l_{1}\right)^{L}=e^{i p_{1} L}=1 .
$$

Assuming that $l_{1} \neq 1$ we can substitute this into (4.4), and we see that the overlap vanishes for all on-shell states with $l_{1} \neq 1$. However, we will be interested in the on-shell states with non-vanishing overlap, therefore we need to consider the case $l_{1}=1$.

In this simple one-particle problem the summation for the exceptional case $l_{1}=1$ is rather trivial, and already given in (4.4). However, in order to get experience for the more complicate cases we also derive this using a limiting procedure: we use the continuity of the scalar product, and investigate the $l_{1} \rightarrow 1$ limit of the $l_{1} \neq 1$ case of (4.4). This gives

$$
\left\langle X_{F} \mid \lambda_{1}=0\right\rangle=\lim _{l_{1} \rightarrow 0} \frac{a_{1}-1}{l_{1}-1}=\lim _{p_{1} \rightarrow 0} \frac{e_{1}^{i p_{1} L}-1}{e^{i p_{1}}-1}=L,
$$

where we used the definition of the $a$ - and $l$-variables.

Even though this is a trivial example, it already highlights a crucial observation: having computed a generic off-shell overlap, the operations of "substituting the Bethe equations" and "taking the limit towards the parity invariant states" do not commute, and it is important to perform the second step first.

\subsection{Two-particle states}

We now consider the two-particle case. The structure of the overlaps of the integrable boundary state and two-particle states has been studied in [4], where the role of the apparent pole (to be discussed below) was explained.

In this case the overlap is given by the summation

$$
\left\langle X_{F} \mid \lambda_{1}, \lambda_{2}\right\rangle=f\left(l_{2}, l_{1}\right) \sum_{0 \leq x_{1}<x_{2} \leq L-1} l_{1}^{x_{1}} l_{2}^{x_{2}}+f\left(l_{1}, l_{2}\right) \sum_{0 \leq x_{1}<x_{2} \leq L-1} l_{2}^{x_{1}} l_{1}^{x_{2}} .
$$

Let us now introduce the function

$$
B_{2}\left(l_{1}, l_{2} \mid L\right)=\sum_{0 \leq x_{1}<x_{2} \leq L-1} l_{1}^{x_{1}} l_{2}^{x_{2}} .
$$


Assuming that

$$
l_{1} \neq 1, \quad l_{2} \neq 1, \quad l_{1} l_{2} \neq 1
$$

we can perform the summation explicitly, yielding

$$
B_{2}\left(l_{1}, l_{2} \mid L\right)=\frac{\left(l_{1} l_{2}\right)^{L}-1}{\left(l_{1} l_{2}-1\right)\left(l_{1}-1\right)}-\frac{l_{2}^{L}-1}{\left(l_{2}-1\right)\left(l_{1}-1\right)} .
$$

Substituting this back into (4.7) and the introducing the $a$-variables the overlap can be written as

$$
\begin{aligned}
\left\langle X_{F} \mid \lambda_{1}, \lambda_{2}\right\rangle= & f\left(l_{2}, l_{1}\right)\left[\frac{a_{1} a_{2}-1}{\left(l_{1} l_{2}-1\right)\left(l_{1}-1\right)}-\frac{a_{2}-1}{\left(l_{2}-1\right)\left(l_{1}-1\right)}\right] \\
& +f\left(l_{1}, l_{2}\right)\left[\frac{a_{1} a_{2}-1}{\left(l_{1} l_{2}-1\right)\left(l_{2}-1\right)}-\frac{a_{1}-1}{\left(l_{2}-1\right)\left(l_{1}-1\right)}\right] .
\end{aligned}
$$

Let us now substitute the Bethe equations which in this case read

$$
a_{1}=\frac{f\left(l_{2}, l_{1}\right)}{f\left(l_{1}, l_{2}\right)}, \quad a_{2}=\frac{f\left(l_{1}, l_{2}\right)}{f\left(l_{2}, l_{1}\right)} .
$$

It can be seen by direct computation that after substitution we get identically zero! This means that all on-shell overlaps vanish, unless one of the conditions in (4.9) is broken. Note that we did not use the specific form of the function $f\left(l_{1}, l_{2}\right)$ : the vanishing of the overlap follows directly from the functional form of the Bethe wave function.

The non-vanishing overlaps are obtained in the special cases, where $l_{1}=1, l_{2}=1$ or $l_{1} l_{2}=1$. For on-shell states we can not have $l_{1}=1$ or $l_{2}=1$ except for very special cases of fine tuned solutions. On the other hand, the condition

$$
l_{1} l_{2}=e^{i\left(p_{1}+p_{2}\right)}=1
$$

is very natural: this is the requirement for the pair structure in the rapidities!

In order to get the overlaps with $l_{1} l_{2}=1$ we can choose two ways: either we compute the function $B_{2}$ directly for this special case, or we perform the limiting procedure from offshell rapidities to on-shell solutions with $l_{1} l_{2}=1$. We choose the second method because it can be generalized to the multi-particle cases.

If we regard the expression (4.11) as a function of 4 variables $l_{1}, l_{2}$ and $a_{1}, a_{2}$, then it has a pole $1 /\left(l_{1} l_{2}-1\right)$ associated with the pair condition. The overlap itself is a regular function of the original $l$-variables, therefore the residue has to be zero in the physical case, when $a_{j}=l_{j}^{L}$. Collecting the terms for the residue around $l_{1} l_{2}=1$ gives

$$
\left\langle X_{F} \mid \lambda_{1}, \lambda_{2}\right\rangle \sim \frac{a_{1} a_{2}-1}{l_{1} l_{2}-1}\left[\frac{f\left(l_{2}, l_{1}\right)}{l_{1}-1}+\frac{f\left(l_{1}, l_{2}\right)}{l_{2}-1}\right] .
$$

In the physical case $a_{j}=l_{j}^{L}$, and the pre-factor is a finite expression of the type $0 / 0$; its finite value is actually $L$. Now we argue that the finite value of the overlap comes only from this apparent pole: all other contributions to the overlap add up to zero for on-shell states, because they are zero for a generic configuration satisfying (4.9). We thus obtain the exact result for on-shell states with the pair structure:

$$
\left\langle X_{F} \mid \lambda_{1},-\lambda_{1}\right\rangle=L\left[\frac{f\left(l_{2}, l_{1}\right)}{l_{1}-1}+\frac{f\left(l_{1}, l_{2}\right)}{l_{2}-1}\right], \quad \text { with } l_{2}=\frac{1}{l_{1}} .
$$




\subsection{Multi-particle states}

The general strategy for the overlaps will mirror the one-particle case. First we introduce some definitions and auxiliary functions.

We call a set of Bethe rapidities $\boldsymbol{\lambda}_{N}$ zero-free, if there is no subset of $\boldsymbol{\lambda}_{N}$ where the sum of the rapidities is zero. Accordingly, the set $\boldsymbol{l}_{N}$ is zero free, when there is no subset of the $l$-variables such that their product is 1 . States with the pair structure are clearly not zero-free: they are the exceptional states that lead to non-zero overlaps.

Here we investigate overlaps with more general integrable initial states. For simplicity we still restrict ourselves to product states, but we allow for an arbitrary two-site state, thus we consider

$$
|\Psi\rangle=\otimes_{j=1}^{L / 2}|\psi\rangle, \quad|\psi\rangle \in \mathcal{H}_{j} \otimes \mathcal{H}_{j+1} .
$$

In the XXZ chain all two-site states are integrable [10], but in models with higher dimensional local spaces the integrability condition puts a restriction on $|\psi\rangle$. Note that the one-site invariant product state considered above is a special case of such two-site states.

The overlap with the reference state is

$$
\langle\Psi \mid \Omega\rangle=\left(\psi_{00}\right)^{L / 2},
$$

where $\psi_{00}$ denotes the two-site overlap between the initial state and the reference state. In the compact cases it is given by $\psi_{00}=\langle\psi \mid \uparrow \uparrow\rangle$, and in the non-compact case by $\psi_{00}=\langle\psi \mid 00\rangle$.

For simplicity we focus on cases where $\psi_{00} \neq 0$. Furthermore we set the normalization to $\psi_{00}=1$, such that the overlap with the reference state is always 1 . Initial states with $\psi_{00}=0$ can be treated with a limiting procedure, see for example the case of the Néel state below.

We consider the overlaps

$$
\mathcal{S}_{N}\left(\boldsymbol{\lambda}_{N}\right)=\left\langle\Psi \mid \boldsymbol{\lambda}_{N}\right\rangle
$$

with the Bethe states given in (2.30). It follows from the explicit form of the wave function that every such an overlap is a rational function of the $l$-variables. The $L$ dependence is hidden in the summation limits. We will show below that for zero-free sets the summations can be performed explicitly, yielding formulae that only involve the $l_{j}$ and $a_{j}=\left(l_{j}\right)^{L}$ for each $j$, but they do not depend on the volume $L$ in any other way.

Let us therefore introduce the function $\mathcal{S}_{N}\left(\boldsymbol{\lambda}_{N}, \boldsymbol{a}_{N}\right)$, which is obtained after these formal manipulations, and after introducing the $a$-variables:

$$
\mathcal{S}_{N}\left(\boldsymbol{l}_{N}, \boldsymbol{a}_{N}\right)=\left\langle\Psi \mid \boldsymbol{\lambda}_{N}\right\rangle_{\text {summed }} .
$$

Regarded as a function of a total number of $2 N$ variables, this function does not depend on $L$ anymore. It follows from the form of the wave function and the real space summations that these functions can always be written as

$$
\mathcal{S}_{N}\left(\boldsymbol{l}_{N}, \boldsymbol{a}_{N}\right)=\sum_{\sigma \in S_{N}} \prod_{j>k} f\left(l_{\sigma_{j}}, l_{\sigma_{k}}\right) B_{N}\left(\sigma \boldsymbol{l}_{N}, \sigma \boldsymbol{a}_{N}\right),
$$

where $B_{N}$ is the "kinematical" part of the overlap, which arises from a simple real space summation. It depends on the initial state; explicit formulae will be given below. In the 
formula above it is understood that $\sigma \boldsymbol{l}_{N}, \sigma \boldsymbol{a}_{N}$ are the permutations of the corresponding ordered sets, namely

$$
\sigma \boldsymbol{l}_{N}=\left\{l_{\sigma_{1}}, l_{\sigma_{2}}, \cdots, l_{\sigma_{N}}\right\}, \quad \sigma \boldsymbol{a}_{N}=\left\{a_{\sigma_{1}}, a_{\sigma_{2}}, \cdots, a_{\sigma_{N}}\right\} .
$$

The quantity $B_{N}$ for some special cases was already defined and computed in [4]. An analogous computation for a non-integrable overlap was performed recently in [35].

Let us also define the function $\tilde{\mathcal{S}}_{N}\left(\boldsymbol{l}_{N}\right)$ which is obtained from $\mathcal{S}_{N}$ by the formal substitution of the Bethe equations. This means that for each $a_{j}$ we substitute the r.h.s. of the corresponding equation from (2.31). It is clear from the above that $\tilde{\mathcal{S}}_{N}$ is a symmetric rational function of the set $\boldsymbol{l}_{N}$.

Theorem 1. The rational function $\tilde{\mathcal{S}}_{N}\left(\boldsymbol{l}_{N}\right)$ is identically zero.

Proof. The function $\tilde{\mathcal{S}}_{N}$ does not depend on the volume anymore, it only depends on the $l$-variables. In the definition of $\mathcal{S}_{N}$ we assumed that the set of rapidities is zero-free. The zero-free sets can not satisfy the integrability condition, therefore their overlaps have to be zero. This implies, that the function $\tilde{\mathcal{S}}_{N}$ vanishes for all those sets $\boldsymbol{l}_{N}$ that are zero-free solutions to the Bethe equations for any volume. This means that the rational function $\tilde{\mathcal{S}}_{N}$ vanishes at an infinite number of points, therefore it is identically zero.

The non-vanishing overlaps are obtained from $\mathcal{S}_{N}$ by a limiting procedure similar to the two-particle case detailed above. The key observation is that for each pair of rapidities (or $l$-variables $l_{j}, l_{k}$ ) there is an apparent simple pole of $S_{N}$, which is proportional to

$$
\frac{a_{j} a_{k}-1}{l_{j} l_{k}-1} .
$$

In the physical cases, when the $a$-variables are actually given by $a_{j}=\left(l_{j}\right)^{L}$, such a factor simply produces $L$. However, it is important that we can substitute the Bethe equation only after these pole contributions are correctly evaluated. Furthermore, all non-zero terms in the overlap can only come from such terms, because if we substitute the Bethe equations before the limit, we get zero identically.

Now we compute $\mathcal{S}_{N}$ for paired rapidities. We regard $\boldsymbol{l}_{N}$ and $\boldsymbol{a}_{N}$ as independent variables in the intermediate steps of the computation. We can still assume that there is a well-defined function $a(l)$ connecting the $l$ - and $a$-variables, but we do not require the relation $a(l)=l^{L}$ anymore. We will see below that a recursive computation of the overlaps will require to treat more general $a(l)$ functions.

We will consider the limit

$$
l_{2 j-1} l_{2 j} \rightarrow 1, \quad a_{2 j-1} a_{2 j} \rightarrow 1, \quad j=1, \ldots, N / 2 .
$$

Let us now investigate the apparent pole at say $l_{1} l_{2}=1$.

Proposition 1. The formal pole of $\mathcal{S}_{N}$ around the point $l_{1} l_{2}=1$ is of the form

$$
\mathcal{S}_{N}(L) \sim \frac{a_{1} a_{2}-1}{l_{1} l_{2}-1} F\left(\lambda_{1}\right) \prod_{j=3}^{N} f\left(\lambda_{1}-\lambda_{j}\right) f\left(-\lambda_{1}-\lambda_{j}\right) \mathcal{S}_{N-2}^{\bmod }(\not 1, \not 2, L),
$$


where $\mathcal{S}_{N-2}^{\text {mod }}$ is the formal overlap for $N-2$ particles not including 1 and 2, evaluated with the following modified a-variables:

$$
a_{j}^{\bmod }=\frac{f\left(l_{j}, l_{1}\right)}{f\left(l_{1}, l_{j}\right)} \frac{f\left(l_{j}, 1 / l_{1}\right)}{f\left(1 / l_{1}, l_{j}\right)} a_{j} .
$$

In (4.24) $F(\lambda)$ is a rational function which carries the dependence on the initial state.

At present we do not have a general proof of this statement. However, we are able to rigorously prove it in concrete cases. This leads to the determination of the function $F(\lambda)$. Examples for this will be shown in the next section.

Eq. (4.24) can be considered as a recursion relation for the overlaps. It is rather similar to the recursion relations for scalar products of Bethe states [17] or form factors [36, 37] (see also $[38,39])$. In fact, the modification rule above is a rather straightforward generalization of a similar rule for scalar products, first derived by Korepin in [17]. However, the origin of the poles is different: in the previous cases in the literature the singularities are the so-called kinematical poles of the scalar products or form factors, which appear when two rapidities in the bra and ket vectors approach each other. On the other hand, here the two rapidities responsible for the pole are within the same Bethe vector, and the apparent singularity is associated with the pair structure. The role of such apparent poles was first recognized in [4], and has been used in [35] to study the large $L$ behaviour of the overlaps.

It is important that if the original $l$ - and $a$-variables satisfy the Bethe equations, then the restricted set of $l$-variables is still on-shell with respect to the modified $a$-variables.

We now investigate the limit of the paired rapidities on the basis of the above recursion relation. Let us therefore introduce the set of "positive" rapidities $\boldsymbol{\lambda}_{N / 2}^{+}$, such that the paired limit is taken as

$$
\lambda_{2 j-1} \rightarrow \lambda_{j}^{+}, \quad \lambda_{2 j} \rightarrow-\lambda_{j}^{+}, \quad j=1 \ldots N .
$$

Similar notations are understood for the $l$ - and $a$-variables.

For future use we introduce one more set of variables which will play an important role. For each $j=1 \ldots N / 2$ we define

$$
m_{j}=m\left(\lambda_{j}\right)=-\left.i \frac{d}{d \lambda} \log (a(\lambda))\right|_{\lambda=\lambda_{j}} .
$$

In the original physical case $a_{j}=l_{j}^{L}=e^{i p\left(\lambda_{j}\right) L}$ we have $m_{j}=p^{\prime}\left(\lambda_{j}\right) L$, but generally we will treat the $m$-variables as independent.

Let us define the function $D\left(\boldsymbol{\lambda}_{N / 2}^{+}, \boldsymbol{m}_{N / 2}^{+}\right)$as the limit of the function $S_{N}$ described by (4.26). This is a symmetric function under a simultaneous permutation of its variables. It is a rational function of $\boldsymbol{\lambda}_{N / 2}^{+}$and it is at most linear in each of the $m$-variables. The latter property follows from the fact that $S_{N}$ has only single poles associated to each pair.

Theorem 2. The function $D$ satisfies the recursion

$$
\frac{\partial D\left(\boldsymbol{\lambda}_{N / 2}^{+}, \boldsymbol{m}_{N / 2}^{+} \mid L\right)}{\partial m_{1}^{+}}=\frac{F\left(\lambda_{1}^{+}\right)}{p^{\prime}\left(\lambda_{1}^{+}\right)} \prod_{l=2}^{N / 2} \bar{f}\left(\lambda_{1}^{+}, \lambda_{l}^{+}\right) \times D\left(\boldsymbol{\lambda}_{N / 2-1}^{+}, \boldsymbol{m}_{N / 2-1}^{+, \bmod } \mid L\right),
$$


where we defined the modification rule for the m-parameters

$$
m_{\bmod }(\lambda)=m(\lambda)+\varphi^{+}\left(\lambda, \lambda_{1}^{+}\right) .
$$

Proof. This follows immediately from (4.24), using also Theorem 1. The modification rule for the $m$-variables follows from

$$
m_{\bmod }(\lambda)=-i \frac{d}{d \lambda} \log \left(a_{\bmod }(\lambda)\right)
$$

and using (4.25) we get (4.29).

Theorem 3. The solution of the recursion (4.28) is

$$
D\left(\boldsymbol{\lambda}_{N / 2}^{+}, \boldsymbol{m}_{N / 2}^{+} \mid L\right)=\prod_{j=1}^{N / 2} \frac{F\left(\lambda_{j}^{+}\right)}{p^{\prime}\left(\lambda_{j}^{+}\right)} \prod_{1 \leq j<k \leq N / 2} \bar{f}\left(\lambda_{j}^{+}, \lambda_{k}^{+}\right) \times \operatorname{det} G_{N / 2}^{+} .
$$

Proof. Our proof follows the method of Korepin derived originally for the Gaudin determinant describing the norm of the Bethe states [17].

First we define a function $\tilde{D}\left(\boldsymbol{\lambda}_{N / 2}^{+}, \boldsymbol{m}_{N / 2}^{+}\right)$through

$$
D\left(\boldsymbol{\lambda}_{N / 2}^{+}, \boldsymbol{m}_{N / 2}^{+} \mid L\right)=\prod_{j=1}^{N / 2} \frac{F\left(\lambda_{j}^{+}\right)}{p^{\prime}\left(\lambda_{j}^{+}\right)} \prod_{1 \leq j<k \leq N / 2} \bar{f}\left(\lambda_{j}^{+}, \lambda_{k}^{+}\right) \tilde{D}\left(\boldsymbol{\lambda}_{N / 2}^{+}, \boldsymbol{m}_{N / 2}^{+} \mid L\right) .
$$

It follows from (4.28) that the linear parts in $m_{j}^{+}$is given by

$$
\frac{\partial \tilde{D}\left(\boldsymbol{\lambda}_{N / 2}^{+}, \boldsymbol{m}_{N / 2}^{+} \mid L\right)}{\partial m_{j}^{+}}=\tilde{D}\left(\boldsymbol{\lambda}_{N / 2-1}^{+}, \boldsymbol{m}_{N / 2-1}^{+, \bmod } \mid L\right)
$$

where it is understood that $m_{j}^{+}$is not included in the arguments on the r.h.s. and the modification rule is given by (4.29).

The function $\tilde{D}$ satisfies the following properties:

- It is symmetric in all its variables.

- It is at most linear in each $m_{j}$.

- It is zero if all $m_{j}=0$.

- The linear piece in each $m_{j}$ is given by (4.33).

It is easy to see that the unique solution for this linear recursion with the given properties is

$$
\tilde{D}=\operatorname{det} \tilde{G}_{N / 2}, \quad \tilde{G}_{j k}=\delta_{j k}\left[m_{j}^{+}+\sum_{l=1}^{N / 2} \varphi^{+}\left(\lambda_{j}^{+}, \lambda_{l}^{+}\right)\right]-\varphi^{+}\left(\lambda_{j}^{+}, \lambda_{k}^{+}\right) .
$$

In the physical case we need to set $m_{j}^{+}=p^{\prime}\left(\lambda_{j}^{+}\right) L$. 
The normalized squared overlap is obtained after dividing by the norm (3.60). Using the factorization (3.57) we eventually obtain

$$
\frac{\left|\left\langle\Psi \mid \boldsymbol{\lambda}_{N / 2}^{+}\right\rangle\right|^{2}}{\left\langle\boldsymbol{\lambda}_{N / 2}^{+} \mid \boldsymbol{\lambda}_{N / 2}^{+}\right\rangle}=\left[\prod_{j=1}^{N / 2} \frac{\left|F\left(\lambda_{j}^{+}\right)\right|^{2}}{f\left(2 \lambda_{j}^{+}\right) f\left(-2 \lambda_{j}^{+}\right)}\right] \frac{\operatorname{det} G^{+}}{\operatorname{det} G^{-}} .
$$

The single particle overlap function is thus determined by the function $F(\lambda)$ which determines the apparent singularity of the off-shell overlap:

$$
u(\lambda)=\frac{\left|F\left(\lambda_{j}^{+}\right)\right|^{2}}{f\left(2 \lambda_{j}^{+}\right) f\left(-2 \lambda_{j}^{+}\right)} .
$$

With this we have finished outlining our general strategy. What remains to be proven is the fundamental singularity relation (4.24), together with finding the function $F(\lambda)$ in specific cases. This is presented in the next section.

\section{Exact overlap formulae - Concrete cases}

\subsection{The state $\left|X_{F}\right\rangle$ in the Heisenberg chains}

Here we consider the state $\left|X_{F}\right\rangle$ defined in (4.1). Now the overlap can be written as (4.20) with the $B$-function given by

$$
B_{N}\left(l_{1}, l_{2}, \ldots, l_{N} \mid L\right)=\sum_{x_{1}=0}^{L-N} \sum_{x_{2}=x_{1}+1}^{L-N+1} \cdots \sum_{x_{N}=x_{N-1}+1}^{L-1} l_{1}^{x_{1}} l_{2}^{x_{2}} \ldots l_{N}^{x_{N}} .
$$

Note that the positions of the particles go from 0 to $L-1$.

Regarding the first function we get

$$
B_{1}\left(l_{1} \mid L\right)=\frac{l_{1}^{L}-1}{l_{1}-1} .
$$

The second function is determined by the simple difference equation

$$
B_{2}(L)-B_{2}(L-1)=l_{2}^{L-1} B_{1}(L-1),
$$

which can be derived from the definition (5.1). In fact, we have the following general recursion relation

$$
B_{N}(L)-B_{N}(L-1)=l_{N}^{L-1} B_{N-1}(L-1) .
$$

The proof of this recursion relation is as follows. Consider the chain of length $L$ with $N$ particles, the corresponding quantity is $B_{N}(L)$. Note that from (5.1), $B_{N}(L)$ can be written as the sum of two parts, corresponding to whether the last site is occupied or not. When the last site is empty, all particles sit in the first $L-1$ sites, the contribution is given by $B_{N}(L-1)$. When the last site is occupied, since the particle is ordered and the site can be occupied by at most one particle, it must be the particle with $l_{N}$ which occupies the last 
site. The contribution from this particle is $l_{N}^{L-1}$. The rest $N-1$ particles are distributed in the first $L-1$ sites whose contribution is given by $B_{N-1}(L-1)$. This implies (5.4). This recursion relation is very helpful for deriving a closed form formula for $B_{N}(L)$, as we will show below.

Substituting (5.2) into (5.3) we get

$$
B_{2}(L)-B_{2}(L-1)=l_{2}^{L-1} \frac{l_{1}^{L-1}-1}{l_{1}-1}=\frac{\left(l_{1} l_{2}\right)^{L-1}-l_{2}^{L-1}}{l_{1}-1} .
$$

The solution to this recursion is

$$
B_{2}(L)=\frac{\left(l_{1} l_{2}\right)^{L}}{\left(l_{1} l_{2}-1\right)\left(l_{1}-1\right)}-\frac{l_{2}^{L}-1}{\left(l_{2}-1\right)\left(l_{1}-1\right)}+C,
$$

where $C$ is an $L$ independent integration constant. It can be fixed easily by computing $B_{2}(L)$ at $L=2$, which is simply

$$
B_{2}(2)=l_{2}
$$

This fixes $C$ and we get

$$
B_{2}(L)=\frac{\left(l_{1} l_{2}\right)^{L}}{\left(l_{1} l_{2}-1\right)\left(l_{1}-1\right)}-\frac{l_{2}^{L}}{\left(l_{2}-1\right)\left(l_{1}-1\right)}+\frac{l_{2}}{\left(l_{2}-1\right)\left(l_{1} l_{2}-1\right)} .
$$

We can continue along these lines for $N=3$. The recursion relation reads

$$
B_{3}(L)-B_{3}(L-1)=l_{3}^{L-1} B_{2}(L-1) .
$$

Solving this with the appropriate initial condition $B_{3}(3)=l_{2} l_{3}^{2}$ we get

$$
\begin{aligned}
B_{3}(L)= & \frac{\left(l_{1} l_{2} l_{3}\right)^{L}}{\left(l_{1} l_{2} l_{3}-1\right)\left(l_{1} l_{2}-1\right)\left(l_{1}-1\right)}-\frac{\left(l_{2} l_{3}\right)^{L}}{\left(l_{2} l_{3}-1\right)\left(l_{2}-1\right)\left(l_{1}-1\right)}+ \\
& +\frac{l_{3}^{L} l_{2}}{\left(l_{3}-1\right)\left(l_{2}-1\right)\left(l_{1} l_{2}-1\right)}-\frac{l_{2} l_{3}^{2}}{\left(l_{1} l_{2} l_{3}-1\right)\left(l_{2} l_{3}-1\right)\left(l_{3}-1\right)} .
\end{aligned}
$$

Continuing this for the general $N$-particle case we get

$$
B_{N}(L)=\sum_{j=0}^{N} \frac{(-1)^{j}\left(\prod_{k=j+1}^{N} l_{k}\right)^{L} \prod_{k=2}^{j} l_{k}^{k-1}}{\prod_{k=j+1}^{N}\left(\prod_{o=j+1}^{k} l_{o}-1\right) \times \prod_{k=1}^{j}\left(\prod_{o=k}^{j} l_{o}-1\right)},
$$

where we also used the general initial condition

$$
B_{N}(N)=l_{2} l_{3}^{2} \ldots l_{N}^{N-1}
$$

Substituting the $a$-variables leads to

$$
B_{N}\left(\left\{a_{j}\right\},\left\{l_{j}\right\}, L\right)=\sum_{j=0}^{N} \frac{(-1)^{j} \prod_{k=j+1}^{N} a_{k} \prod_{k=2}^{j} l_{k}^{k-1}}{\prod_{k=j+1}^{N}\left(\prod_{o=j+1}^{k} l_{o}-1\right) \times \prod_{k=1}^{j}\left(\prod_{o=k}^{j} l_{o}-1\right)}
$$

This will be the ingredient function for the overlaps, which will have a summation over permutations, and multiplication with factors related to the $S$-matrix. We emphasize that the $L$ dependence is now all hidden in $\left\{a_{j}\right\}$ in the final expression (5.13) and no longer appears in the limits of the summations. This manipulation makes it possible to impose the Bethe equations. The formula (5.13) was first computed in [4]. 


\subsubsection{Determining the singular piece}

Now we intend to compute the residue of the pole $1 /\left(l_{1} l_{2}-1\right)$ of $\mathcal{S}_{N}$. The overlap itself is given by $N$ ! terms, but from the actual form of the $B$-function it can be seen that the desired pole will only be present in those permutations that put the particles 1 and 2 to neighboring positions. This is equivalent to the statement that $B_{N}$ has a pole of the form $1 /\left(l_{j} l_{k}-1\right)$ if $|j-k|=1$.

Let us therefore pick some number $m$ and investigate the residue

$$
\operatorname{Res}_{l_{m} l_{m+1} \rightarrow 1} B_{N}(L) .
$$

To this order we write the $B$-function as

$$
B_{N}(L)=\sum_{j=0}^{N} B_{N, j}(L)
$$

with

$$
B_{N, j}(L)=\frac{(-1)^{j} \prod_{k=j+1}^{N} a_{k} \prod_{k=2}^{j} l_{k}^{k-1}}{\prod_{k=j+1}^{N}\left(\prod_{o=j+1}^{k} l_{o}-1\right) \times \prod_{k=1}^{j}\left(\prod_{o=k}^{j} l_{o}-1\right)} .
$$

Let us look at the poles of the type $1 /\left(l_{m} l_{m+1}-1\right)$. There are two singular pieces given by $B_{N, m-1}$ and $B_{N, m+1}$, and their sum reads

$$
\begin{aligned}
& \frac{(-1)^{m-1} \prod_{k=m}^{N} a_{k} \prod_{k=2}^{m-1} l_{k}^{k-1}}{\prod_{k=m}^{N}\left(\prod_{o=m}^{k} l_{o}-1\right) \times \prod_{k=1}^{m-1}\left(\prod_{o=k}^{m-1} l_{o}-1\right)} \\
& \quad+\frac{(-1)^{m-1} \prod_{k=m+2}^{N} a_{k} \prod_{k=2}^{m+1} l_{k}^{k-1}}{\prod_{k=m+2}^{N}\left(\prod_{o=m+2}^{k} l_{o}-1\right) \times \prod_{k=1}^{m+1}\left(\prod_{o=k}^{m+1} l_{o}-1\right)} \\
& =\frac{(-1)^{m-1} a_{m} a_{m+1} \prod_{k=m+2}^{N} a_{k} \prod_{k=2}^{m-1} l_{k}^{k-1}}{\left(l_{m}-1\right)\left(l_{m} l_{m+1}-1\right) \prod_{k=m+2}^{N}\left(\prod_{o=m}^{k} l_{o}-1\right) \times \prod_{k=1}^{m-1}\left(\prod_{o=k}^{m-1} l_{o}-1\right)} \\
& \quad+\frac{(-1)^{m-1}\left(l_{m} l_{m+1}\right)^{m} l_{m+1} \prod_{k=m+2}^{N} a_{k} \prod_{k=2}^{m-1} l_{k}^{k-1}}{\prod_{k=m+2}^{N}\left(\prod_{o=m+2}^{k} l_{o}-1\right) \times\left(l_{m} l_{m+1}-1\right)\left(l_{m+1}-1\right) \prod_{k=1}^{m-1}\left(\prod_{o=k}^{m+1} l_{o}-1\right)}
\end{aligned}
$$

So altogether the singular piece in $B_{N}$ is

$$
\begin{aligned}
B_{N}\left(\left\{a_{j}\right\},\left\{l_{j}\right\}, L\right) \sim & \frac{a_{m} a_{m+1}-1}{l_{m} l_{m+1}-1} \\
& \times \frac{1}{l_{m}-1} \frac{(-1)^{m-1} \prod_{k=m+2}^{N} a_{k} \prod_{k=2}^{m-1} l_{k}^{k-1}}{\prod_{k=m+2}^{N}\left(\prod_{o=m}^{k} l_{o}-1\right) \times \prod_{k=1}^{m-1}\left(\prod_{o=k}^{m-1} l_{o}-1\right)},
\end{aligned}
$$

which can be written as

$$
B_{N}\left(\left\{a_{j}\right\},\left\{l_{j}\right\}, L\right) \sim \frac{a_{m} a_{m+1}-1}{l_{m} l_{m+1}-1} \frac{1}{l_{m}-1} B_{N-2, m-1}(\{1,2, \ldots, \not \varkappa, m \subset-\ldots, N\}, L) .
$$


In order to determine the singularity of $\mathcal{S}_{N}$ we need to sum over all permutations that put the particles 1 and 2 to neighboring positions and multiply with the $f$-functions corresponding to the permutations. It is important that once we pick positions $m$ and $m+1$ there are still two possibilities corresponding to the relative ordering of particles 1 and 2 . These two terms will have many common factors for each $m$, and the sum of those factors which are different is

$$
F\left(\lambda_{m}, \lambda_{m+1}\right)=\frac{f\left(\lambda_{m+1}-\lambda_{m}\right)}{l_{m}-1}+\frac{f\left(\lambda_{m}-\lambda_{m+1}\right)}{l_{m+1}-1} .
$$

Using the symmetry we can introduce

$$
F(\lambda)=\frac{f(-2 \lambda)}{l(\lambda)-1}+\frac{f(2 \lambda)}{l(-\lambda)-1} .
$$

The remaining additional $f$-factors for these terms will be

$$
\prod_{j=1}^{m-1} f\left(\lambda_{m}-\lambda_{j}\right) f\left(\lambda_{m+1}-\lambda_{j}\right) \prod_{j=m+2}^{N} f\left(\lambda_{j}-\lambda_{m}\right) f\left(\lambda_{j}-\lambda_{m+1}\right) .
$$

This can be written in the form

$$
\prod_{\substack{j=1 \\ j \neq m, m+1}}^{N} f\left(\lambda_{m}-\lambda_{j}\right) f\left(\lambda_{m+1}-\lambda_{j}\right) \times \prod_{j=m+2}^{N} \frac{f\left(\lambda_{j}-\lambda_{m}\right)}{f\left(\lambda_{m}-\lambda_{j}\right)} \frac{f\left(\lambda_{j}-\lambda_{m+1}\right)}{f\left(\lambda_{m+1}-\lambda_{j}\right)} .
$$

Note that ratios of $f$-functions appear such that they multiply the $a$-variables in a well defined way, namely the residue can be formulated by introducing the modification rule

$$
a_{j}^{\text {mod }}=\frac{f\left(\lambda_{j}-\lambda_{m}\right)}{f\left(\lambda_{m}-\lambda_{j}\right)} \frac{f\left(\lambda_{j}-\lambda_{m+1}\right)}{f\left(\lambda_{m+1}-\lambda_{j}\right)} a_{j} .
$$

It is important that if the original set $\boldsymbol{\lambda}_{N}$ satisfies the original Bethe equations, then the set $\boldsymbol{\lambda}_{N} \backslash\left\{\lambda_{m}, \lambda_{m+1}\right\}$ satisfies the Bethe equations with the modified $a$-parameters.

Summing over all remaining permutations, altogether the singularity of the overlap at $l_{1} l_{2}=1$ is

$$
\mathcal{S}_{N}(L) \sim \frac{a_{1} a_{2}-1}{l_{1} l_{2}-1} F\left(\lambda_{1}\right) \prod_{j=3}^{N} f\left(\lambda_{1}-\lambda_{j}\right) f\left(-\lambda_{1}-\lambda_{j}\right) \mathcal{S}_{N-2}^{\bmod }(\not 1, \not 2, L)
$$

with $F(\lambda)$ given by (5.21).

In the XXX model the functions $l(\lambda)$ and $f(\lambda)$ are given by (2.23). Substituting them into (5.21) we obtain $F(\lambda)=0$. This means that all overlaps with $N \neq 0$ are zero. This is in agreement with the fact that any ferromagnetic state is an eigenstate of the Hamiltonian, which lies in the $\mathrm{SU}(2)$ multiplet of the reference state. The overlaps of these states with any Bethe states are identically zero.

In the XXZ model the functions $l(\lambda)$ and $f(\lambda)$ are given by (2.22). This leads to

$$
F(\lambda)=\frac{\sin (\lambda+i \eta / 2) \sin (\lambda-i \eta / 2)}{\cos ^{2}(\lambda)} .
$$


Computing the overlap pre-factor as given by (4.36) we get

$$
u(\lambda)=\frac{F^{2}(\lambda)}{f(2 \lambda) f(-2 \lambda)}=\tan ^{2}(\lambda) \tan (\lambda+i \eta / 2) \tan (\lambda-i \eta / 2) .
$$

This coincides with the result obtained in [11], see eq. (3.18) there.

\subsection{Néel and generalized Néel states in the Heisenberg chains}

Let us now consider the boundary state

$$
\left|N_{\alpha}\right\rangle=\otimes_{j=1}^{L / 2}\left(\left(\begin{array}{l}
1 \\
\alpha
\end{array}\right) \otimes\left(\begin{array}{l}
1 \\
0
\end{array}\right)\right) .
$$

This state satisfies the requirement $\psi_{11}=1$, and for non-zero $\alpha$ it has finite overlaps with all parity-invariant Bethe states. In the $\alpha \rightarrow \infty$ limit it turns into the Néel state after re-scaling. It is our intention here to derive the overlaps, and also to show that in the $\alpha \rightarrow \infty$ limit only the states with $N=L / 2$ can have non-zero overlaps.

Now particles can only occupy every odd site. As a result, the computation of the kinematical sum is almost the same as in the previous case, except that now the propagation of particles is restricted to an even number of hoppings. As an effect, the kinematical $B_{N^{-}}$ function is formally the same as before, except for the replacement $L \rightarrow L / 2$ and $l_{j} \rightarrow l_{j}^{2}$ for each $j=1, \ldots, N$. Also, the overlap receives an overall factor of $\alpha^{N}$. As an effect of these changes, instead of the direct pole of the type $1 /\left(l_{1} l_{2}-1\right)$ we obtain poles

$$
\frac{1}{l_{1}^{2} l_{2}^{2}-1}=\frac{1}{l_{1} l_{2}-1} \frac{1}{l_{1} l_{2}+1} .
$$

It can be seen that the residue at $l_{1} l_{2}=1$ gets an extra factor of $1 / 2$. Putting these modifications together we can extract the $F$-function as

$$
F(\lambda)=\frac{\alpha^{2}}{2}\left[\frac{f(-2 \lambda)}{l^{2}(\lambda)-1}+\frac{f(2 \lambda)}{l^{2}(-\lambda)-1}\right]
$$

In the XXX model the substitution of (2.23) leads to

$$
F(\lambda)=\alpha^{2} \frac{u^{2}+1 / 4}{4 u^{2}}
$$

Altogether the one-particle overlap function with the un-normalized state becomes

$$
u(\lambda)=\frac{F^{2}(\lambda)}{f(2 \lambda) f(-2 \lambda)}=\alpha^{4} \frac{u^{2}+1 / 4}{16 u^{2}} .
$$

In order to obtain the overlaps with the Néel state we need to perform the limit $\alpha \rightarrow \infty$ after re-scaling by $\alpha^{L}$. It follows immediately that only the overlaps with $N=L / 2$ survive, as expected.

The resulting overlap formula agrees with the earlier results $[4,7,32]$. 


\subsection{Generalized Néel state in the $\operatorname{SL}(2, \mathbb{R})$ chain}

Let us first consider the overlap with the generalization of $\left|X_{F}\right\rangle$, namely a one-site invariant state

$$
\left|X_{F}\right\rangle=\otimes_{j=1}^{L}\left(\begin{array}{c}
1 \\
1 \\
\vdots
\end{array}\right)=\otimes_{j=1}^{L}\left(e^{S_{+}}|0\rangle\right)=e^{\mathcal{S}_{+}}|\Omega\rangle
$$

This state was already introduced in section 3.1 as the special case of the generalized Néel state $\mid$ Néel $\left._{1}\right\rangle$. This vector belongs to the multiplet of the reference state, so the overlaps with the Bethe states will vanish, in accordance with relation (3.35) for $\kappa=1$. However, it is useful to compute the associated kinematical functions, which can be used later for general $\kappa$.

The overlap with $\left|X_{F}\right\rangle$ is given by the same form as in (4.20) but now the kinematical sum is

$$
B_{N}\left(l_{1}, l_{2}, \ldots, l_{N} \mid L\right)=\sum_{x_{1}=0}^{L-1} \sum_{x_{2}=x_{1}}^{L-1} \ldots \sum_{x_{N}=x_{N-1}}^{L-1} l_{1}^{x_{1}} l_{2}^{x_{2}} \ldots l_{N}^{x_{N}} .
$$

The difference from the compact XXZ model is that now an arbitrary number of particles can occupy the same site, and this changes the summation limits.

In the one-particle case we get the same formula as before:

$$
B_{1}\left(l_{1} \mid L\right)=\frac{l_{1}^{L}-1}{l_{1}-1} .
$$

For $N=2$ the relevant recursion relation is

$$
B_{2}(L)-B_{2}(L-1)=l_{1} \frac{\left(l_{1} l_{2}\right)^{L-1}}{l_{1}-1}-\frac{l_{2}^{L-1}}{l_{1}-1} .
$$

The initial condition is $B_{2}(1)=1$. The solution satisfying this condition is

$$
B_{2}(L)=l_{1} \frac{\left(l_{1} l_{2}\right)^{L}}{\left(l_{1} l_{2}-1\right)\left(l_{1}-1\right)}-\frac{l_{2}^{L}}{\left(l_{1}-1\right)\left(l_{2}-1\right)}+\frac{1}{\left(l_{1} l_{2}-1\right)\left(l_{2}-1\right)} .
$$

Regarding the general multi-particle case the difference equation is

$$
B_{N}(L)-B_{N}(L-1)=l_{N}^{L-1} B_{N-1}(L)
$$

with the initial condition

$$
B_{N}(1)=1
$$

The general solution is

$$
B_{N}(L)=\sum_{j=0}^{N} \frac{(-1)^{j} \prod_{k=j+1}^{N} l_{k}^{L} l_{k}^{N-k}}{\prod_{k=j+1}^{N}\left(\prod_{o=j+1}^{k} l_{o}-1\right) \times \prod_{k=1}^{j}\left(\prod_{o=k}^{j} l_{o}-1\right)} .
$$

The analysis of the singularity of $B_{N}$ can be performed in a similar way as before. We get the relation

$$
B_{N}\left(\left\{a_{j}\right\},\left\{l_{j}\right\}, L\right) \sim \frac{a_{m} a_{m+1}-1}{l_{m} l_{m+1}-1} \frac{l_{m}}{l_{m}-1} B_{N-2, m-1}(\{1,2, \ldots, \not \varkappa, m \subset-\ldots, N\}, L) .
$$


The only change compared to (5.19) is the appearance of an extra factor of $l_{m}$. Completing the computation we obtain the $F$-function as

$$
\begin{aligned}
F(\lambda) & =\frac{f(-2 \lambda) l(\lambda)}{l(\lambda)-1}+\frac{f(2 \lambda) l(-\lambda)}{l(-\lambda)-1} \\
& =\frac{f(-2 \lambda)}{1-l(-\lambda)}+\frac{f(2 \lambda)}{1-l(\lambda)} .
\end{aligned}
$$

In the $\mathrm{SL}(2, \mathbb{R})$ case the corresponding functions are given by (2.24). Substituting them into (5.42) we get $F(\lambda)=0$ as expected.

Now we consider the generalized Néel state $\mid$ Néel $\left._{0}\right\rangle$. The difference is once again that we need to perform the change $l_{j} \rightarrow l_{j}^{2}$. This leads eventually to

$$
F(\lambda)=\frac{1}{2}\left[\frac{f(-2 \lambda)}{1-l^{2}(-\lambda)}+\frac{f(2 \lambda)}{1-l^{2}(\lambda)}\right]
$$

and the overlap function becomes

$$
u(\lambda)=\frac{F^{2}(\lambda)}{f(2 \lambda) f(-2 \lambda)} .
$$

Substituting (2.24) we get the same result as in the XXX case:

$$
u(\lambda)=\frac{F^{2}(\lambda)}{f(2 \lambda) f(-2 \lambda)}=\frac{u^{2}+1 / 4}{16 u^{2}} .
$$

This result agrees with the findings of [1].

\section{Conclusions and discussions}

In this work we derived and proved exact overlap formulae in integrable spin chains. Our method is based on the coordinate Bethe Ansatz representation of the wave functions. The key identity is the singularity property (4.24) of the off-shell overlaps. This is a new result of the present work, which leads to the proof of exact overlaps in a number of cases presented in section 5 .

It is important to compare the present method to the previous derivation of [7], which was the only available rigorous proof before our work. The paper [7] derived the factorized overlaps starting from an exact off-shell determinant formula, based on $[8,9]$ and going back to the work of Tsushiya [16]. This method only works for the boundary states corresponding to the so-called diagonal $K$-matrices. On the other hand, our method is applicable even for off-diagonal $K$-matrices, when there is no determinant formula for the off-shell overlaps.

Nevertheless our method has its drawbacks and limitations. First of all, we were not able to provide a general proof of the relation (4.24), we only proved it on a case by case basis. Clearly, it would be important to find the deeper reason why such a relation holds. Second, our method relies heavily on the coordinate Bethe Ansatz, and therefore it can not be applied in situations where this method fails, for example in models with U(1)symmetry breaking. It would be desirable to study the same problems in more general 
frameworks such as the Separation of Variables (SoV) method. Such a future study might also be helpful for studying overlaps in models solvable by the nested Bethe ansatz, where the present method seems rather cumbersome. We plan to return to this question in future work.

Regarding the interpretation of the factorized overlap formulae let us mention once more the work [15], which treated excited state $g$-functions in integrable QFT. These objects are completely analogous to the finite volume overlaps in the spin chain. In [15] the known structure (3.62) of the overlaps was derived, even before the analogous results for spin chains appeared in the context of the quantum quench. The work [15] compared the computation of certain time-dependent one-point functions in finite and infinite volumes, and derived the correct ratio of determinants using only the density of states for the restricted, parity symmetric configurations. Therefore, [15] provides a rather natural interpretation for the overlaps, much like the parallel observation that the original Gaudin-determinant describes both the density of states and the norm of the Bethe states (see also [40]). It would be desirable to work out the arguments of [15] also in the spin chain situation, and to make them precise. This would complete the understanding of the factorized overlap formulae.

Finally we note that the our method can be applied directly to the Lieb-Liniger model to derive the overlaps with the BEC state, originally found in [41] and proven by a scaling limit of the spin chain in [42].

\section{Acknowledgments}

The work of B.P. was partially supported by the National Research Development and Innovation Office (NKFIH) of Hungary under grant K-16 No. 119204, by the János Bolyai Research Scholarship of the Hungarian Academy of Sciences and the ÚNKP-19-4 New National Excellence Program of the Ministry for Innovation and Technology.

\section{A Formula for coherent states}

In this appendix, we collect some formula for coherent states which are useful in the main text. From the $\mathrm{SL}(2, \mathbb{R})$ algebra and using the formula

$$
e^{A} B e^{-A}=B+[A, B]+\frac{1}{2 !}[A,[A, B]]+\cdots
$$

we can prove the following results

$$
\begin{aligned}
e^{-\alpha S_{+}} S_{-} e^{\alpha S_{+}} & =S_{-}+2 \alpha S_{0}+\alpha^{2} S_{+}, \\
e^{-\alpha S_{+}} S_{0} e^{\alpha S_{+}} & =S_{0}+\alpha S_{+} .
\end{aligned}
$$

Using these relations, we can prove the action of generators on the coherent state

$$
S_{0}|\alpha\rangle=\frac{1}{2}|\alpha\rangle+\alpha S_{+}|\alpha\rangle, \quad S_{-}|\alpha\rangle=\alpha|\alpha\rangle+\alpha^{2} S_{+}|\alpha\rangle .
$$


Open Access. This article is distributed under the terms of the Creative Commons Attribution License (CC-BY 4.0), which permits any use, distribution and reproduction in any medium, provided the original author(s) and source are credited.

\section{References}

[1] Y. Jiang, S. Komatsu and E. Vescovi, Structure constants in $\mathcal{N}=4$ SYM at finite coupling as worldsheet $g$-function, arXiv:1906.07733 [INSPIRE].

[2] Y. Jiang, S. Komatsu and E. Vescovi, Exact three-point functions of determinant operators in planar N = 4 supersymmetric Yang-Mills theory, Phys. Rev. Lett. 123 (2019) 191601 [arXiv: 1907.11242] [INSPIRE].

[3] Z. Bajnok et al., Cylinder partition function of the 6-vertex model from algebraic geometry, arXiv: 2002.09019 [INSPIRE].

[4] M. de Leeuw, C. Kristjansen and K. Zarembo, One-point functions in defect CFT and integrability, JHEP 08 (2015) 098 [arXiv: 1506.06958] [INSPIRE].

[5] I. Buhl-Mortensen, M. de Leeuw, C. Kristjansen and K. Zarembo, One-point functions in AdS/dCFT from matrix product states, JHEP 02 (2016) 052 [arXiv: 1512.02532] [INSPIRE].

[6] M. de Leeuw, C. Kristjansen and S. Mori, AdS/dCFT one-point functions of the SU(3) sector, Phys. Lett. B 763 (2016) 197 [arXiv:1607.03123] [INSPIRE].

[7] M. Brockmann et al., A Gaudin-like determinant for overlaps of Néel and XXZ Bethe states, J. Phys. A 47 (2014) 145003 [arXiv:1401.2877].

[8] B. Pozsgay, Overlaps between eigenstates of the XXZ spin-1/2 chain and a class of simple product states, J. Stat. Mech. 06 (2014) P06011 [arXiv:1309.4593].

[9] K.K. Kozlowski and B. Pozsgay, Surface free energy of the open XXZ spin-1/2 chain, J. Stat. Mech. 052012 P05021.

[10] L. Piroli, B. Pozsgay and E. Vernier, What is an integrable quench?, Nucl. Phys. B 925 (2017) 362 [arXiv: 1709.04796] [INSPIRE].

[11] B. Pozsgay, Overlaps with arbitrary two-site states in the XXZ spin chain, J. Stat. Mech. 1805 (2018) 053103 [arXiv: 1801.03838] [INSPIRE].

[12] M. De Leeuw et al., Spin chain overlaps and the twisted Yangian, JHEP 01 (2020) 176 [arXiv: 1912.09338] [INSPIRE].

[13] B. Pozsgay, L. Piroli and E. Vernier, Integrable matrix product states from boundary integrability, SciPost Phys. 6 (2019) 062 [arXiv:1812.11094] [INSPIRE].

[14] S. Ghoshal and A.B. Zamolodchikov, Boundary $S$ matrix and boundary state in two-dimensional integrable quantum field theory, Int. J. Mod. Phys. A 9 (1994) 3841 [Erratum ibid. A 9 (1994) 4353] [hep-th/9306002] [INSPIRE].

[15] M. Kormos and B. Pozsgay, One-point functions in massive integrable QFT with boundaries, JHEP 04 (2010) 112 [arXiv: 1002.2783] [INSPIRE].

[16] O. Tsuchiya, Determinant formula for the six-vertex model with reflecting end, J. Math. Phys. 39 (1998) 5946 [solv-int/9804010].

[17] V.E. Korepin, Calculation of norms of Bethe wave functions, Commun. Math. Phys. 86 (1982) 391 [INSPIRE]. 
[18] V.M. Braun, S.E. Derkachov and A.N. Manashov, Integrability of three particle evolution equations in QCD, Phys. Rev. Lett. 81 (1998) 2020 [hep-ph/9805225] [INSPIRE].

[19] M. Kirch and A.N. Manashov, Noncompact $\mathrm{SL}(2, \mathbb{R})$ spin chain, JHEP 06 (2004) 035 [hep-th/0405030] [INSPIRE].

[20] L.N. Lipatov, Asymptotic behavior of multicolor QCD at high energies in connection with exactly solvable spin models, JETP Lett. 59 (1994) 596 [hep-th/9311037] [INSPIRE].

[21] L.D. Faddeev and G.P. Korchemsky, High-energy QCD as a completely integrable model, Phys. Lett. B 342 (1995) 311 [hep-th/9404173] [INSPIRE].

[22] V.M. Braun, G.P. Korchemsky and D. Müller, The uses of conformal symmetry in QCD, Prog. Part. Nucl. Phys. 51 (2003) 311 [hep-ph/0306057] [INSPIRE].

[23] R. Frassek, C. Giardinà and J. Kurchan, Non-compact quantum spin chains as integrable stochastic particle processes, arXiv:1904.01048 [INSPIRE].

[24] H. Bethe, Zur Theorie der Metalle, Z. Phys. A 71 (1931) 205.

[25] R. Orbach, Linear antiferromagnetic chain with anisotropic coupling, Phys. Rev. 112 (1958) 309.

[26] L.R. Walker, Antiferromagnetic linear chain, Phys. Rev. 116 (1959) 1089.

[27] C.N. Yang and C.P. Yang, One-dimensional chain of anisotropic spin-spin interactions. I. Proof of bethe's hypothesis for ground state in a finite system, Phys. Rev. 150 (1966) 321.

[28] M.J. Martins and C.S. Melo, Algebraic Bethe ansatz for U(1) invariant integrable models: compact and non-compact applications, Nucl. Phys. B 820 (2009) 620 [arXiv:0902.3476] [INSPIRE].

[29] N. Crampé, E. Ragoucy and L. Alonzi, Coordinate Bethe Ansatz for spin s XXX model, SIGMA 7 (2011) 6 [arXiv:1009.0408].

[30] L.D. Faddeev, How algebraic Bethe ansatz works for integrable model, hep-th/9605187 [INSPIRE].

[31] T. Gombor, private communication.

[32] O. Foda and K. Zarembo, Overlaps of partial Néel states and Bethe states, J. Stat. Mech. 1602 (2016) 023107 [arXiv: 1512.02533] [INSPIRE].

[33] M. De Leeuw, C. Kristjansen and G. Linardopoulos, Scalar one-point functions and matrix product states of AdS/dCFT, Phys. Lett. B 781 (2018) 238 [arXiv:1802.01598] [INSPIRE].

[34] M. Brockmann, J. De Nardis, B. Wouters and J.-S. Caux, Néel-XXZ state overlaps: odd particle numbers and Lieb-Liniger scaling limit, J. Phys. A 47 (2014) 345003 [arXiv: 1403.7469].

[35] M. de Leeuw, C. Kristjansen, and K. E. Vardinghus, A non-integrable quench from AdS/dCFT, Phys. Lett. B 798 (2019) 134940 [arXiv:1906.10714].

[36] F.A. Smirnov, Form-factors in completely integrable models of quantum field theory, Adv. Ser. Math. Phys. 14 (1992) 1 [INSPIRE].

[37] B. Pozsgay, W.-V. van Gerven Oei and M. Kormos, On form factors in nested Bethe Ansatz systems, J. Phys. A 45 (2012) 465007 [arXiv: 1204.4037] [InSPIRE].

[38] Y. Jiang and A. Petrovskii, Diagonal form factors and hexagon form factors, JHEP 07 (2016) 120 [arXiv:1511.06199] [INSPIRE]. 
[39] Y. Jiang, Diagonal form factors and hexagon form factors II. Non-BPS light operator, JHEP 01 (2017) 021 [arXiv:1601.06926] [INSPIRE].

[40] M. Gaudin, B.M. McCoy and T.T. Wu, Normalization sum for the Bethe's hypothesis wave functions of the Heisenberg-Ising chain, Phys. Rev. D 23 (1981) 417.

[41] J. De Nardis, B. Wouters, M. Brockmann and J.S. Caux, Solution for an interaction quench in the Lieb-Liniger Bose gas, Phys. Rev. A 89 (2014) 033601 [arXiv:1308.4310].

[42] M. Brockmann, Overlaps of q-raised Néel states with XXZ Bethe states and their relation to the Lieb-Liniger Bose gas, J. Stat. Mech. 05 (2014) P05006 [arXiv:1402.1471]. 